\title{
The search for failed supernovae with the Large Binocular Telescope: confirmation of a disappearing star
}

\author{
S. M. Adams, ${ }^{1,2 \star ~ C . ~ S . ~ K o c h a n e k, ~}{ }^{2,3}$ J. R. Gerke, ${ }^{2}$ K. Z. Stanek ${ }^{2,3}$ and X. Dai ${ }^{4}$ \\ ${ }^{1}$ Cahill Center for Astrophysics, California Institute of Technology, Pasadena, CA 91125, USA \\ ${ }^{2}$ Department of Astronomy, The Ohio State University, 140 W. 18th Ave., Columbus, OH 43210, USA \\ ${ }^{3}$ Center for Cosmology and AstroParticle Physics (CCAPP), The Ohio State University, 191 W. Woodruff Ave., Columbus, OH 43210, USA \\ ${ }^{4}$ Department of Physics and Astronomy, University of Oklahoma, 440 W. Brooks St, Norman, OK 73019, USA
}

Accepted 2017 March 29. Received 2017 March 28; in original form 2016 September 4

\begin{abstract}
We present Hubble Space Telescope imaging confirming the optical disappearance of the failed supernova (SN) candidate identified by Gerke, Kochanek \& Stanek. This $\sim 25 \mathrm{M}_{\odot}$ red supergiant experienced a weak $\sim 10^{6} \mathrm{~L} \odot$ optical outburst in 2009 and is now at least 5 mag fainter than the progenitor in the optical. The mid-IR flux has slowly decreased to the lowest levels since the first measurements in 2004 . There is faint $\left(2000-3000 \mathrm{~L}_{\odot}\right)$ near-IR emission likely associated with the source. We find the late-time evolution of the source to be inconsistent with obscuration from an ejected, dusty shell. Models of the spectral energy distribution indicate that the remaining bolometric luminosity is $>6$ times fainter than that the progenitor and is decreasing as $\sim t^{-4 / 3}$. We conclude that the transient is unlikely to be an $\mathrm{SN}$ impostor or stellar merger. The event is consistent with the ejection of the envelope of a red supergiant in a failed SN and the late-time emission could be powered by fallback accretion on to a newly formed black hole. Future IR and X-ray observations are needed to confirm this interpretation of the fate for the star.
\end{abstract}

Key words: black hole physics - stars: massive-supernovae: individual: N6946-BH1.

\section{INTRODUCTION}

Supernova (SN) surveys for the deaths of massive stars search for a sudden brightening of a source. However, it is expected that some fraction of massive stars experience a failed SN, forming a black hole without a luminous SN. While this idea is most widely accepted for very high mass stars at lower metallicity (Heger et al. 2003), evidence has recently emerged suggesting that failed SN may also occur in red supergiants (RSGs) with solar metallicity.

First, there is the lack of higher mass SN progenitors, which suggests that higher mass stars may end their lives as failed $\mathrm{SNe}$ (Kochanek et al. 2008). Smartt et al. (2009) and Smartt (2015) more clearly demonstrated that the known progenitors of Type IIP SNe have an upper mass limit of $\lesssim 18 \mathrm{M}_{\odot}$ - well below the expected mass range for RSG at death. Although there are alternative hypotheses for the missing $\mathrm{SN}$ progenitors, such as post-RSG evolution occurring at lower masses (e.g. Smith et al. 2011a; Groh et al. 2013) or enhanced dust formation prior to core collapse (e.g. Walmswell \& Eldridge 2012; Beasor \& Davies 2016), the dearth of higher mass SN progenitors is supported by analyses of stellar populations near SN remnants (Jennings et al. 2014) and the absence of any Type IIP SNe with the nucleosynthetic signatures of higher

\footnotetext{
^E-mail: sma@astro.caltech.edu
}

mass (>20 $\mathrm{M}_{\odot}$ ) progenitors (Jerkstrand et al. 2014). Interestingly, the mass range for failed $\mathrm{SNe}$ suggested by the missing progenitors corresponds to stars with progenitor structures that make them more difficult to explode (O'Connor \& Ott 2011; Ugliano et al. 2012; Pejcha \& Thompson 2015; Ertl et al. 2016; Sukhbold et al. 2016).

Secondly, having a significant fraction of core collapses resulting in failed SNe naturally explains the compact remnant mass function (Kochanek 2014b, 2015). When black holes are formed by 'fall back' on to the protoneutron star during successful SNe, the mass distributions of black holes and neutron stars are continuous because there is no natural mass scale for the amount of fallback (Zhang, Woosley \& Heger 2008; Fryer et al. 2012). Such continuous distributions are inconsistent with observations showing a significant gap between neutron star and black hole masses (Kreidberg et al. 2012; Özel et al. 2012). If the core collapse fails to explode an RSG, the resulting black hole mass is the mass of the progenitor's helium core (Lovegrove \& Woosley 2013), naturally producing the observed gap between neutron star and black hole mass distributions and the observed black hole masses.

Third, there is evidence that the massive star formation rate may exceed the SN rate (Horiuchi et al. 2011, but see Botticella et al. 2012; Xiao \& Eldridge 2015). Finally, the recent detection of gravity waves from a pair of merging black holes with masses of $36_{-4}^{+5}$ and $29_{-4}^{+4} \mathrm{M}_{\odot}$ (Abbott et al. 2016b) likely requires the existence of failed SNe (Abbott et al. 2016a; Belczynski et al. 2016; 
Woosley 2016). These lines of evidence for the existence of failed $\mathrm{SNe}$, while indirect, motivate the direct search for failed SNe.

The formation of a black hole has never been observed and little is known about the range of possible observational signatures. Some stars likely collapse to form black holes without significant transients (Woosley \& Heger 2012). However, a failed SN in an RSG likely leads to a visible transient. Nadezhin (1980) suggested, and Lovegrove \& Woosley (2013) confirmed with hydrodynamic simulations, that the nearly instantaneous loss of gravitational mass through neutrino emission when a core collapses will lead to a hydrodynamic shock capable of unbinding the loosely bound hydrogen envelope of an RSG. The resulting optical signature is a shock breakout with $L \sim 10^{7} \mathrm{~L} \odot$ that lasts for 3-10 d (Piro 2013) followed by a cool $(\sim 3000 \mathrm{~K}), \sim 1$-yr-long, $\sim 10^{6} \mathrm{~L} \odot$ transient powered by the recombination of the unbound envelope (Lovegrove \& Woosley 2013). Once sufficiently cool, the slowly expanding ejecta is an ideal environment for dust formation, but this would only occur after the transient has already begun to fade (Kochanek 2014a). Regardless of the nature of any intervening transient the end result is the disappearance of the progenitor.

Kochanek et al. (2008) proposed a novel survey to monitor the evolved stars in nearby galaxies to search for failed $\mathrm{SNe}$ as disappearing stars. Gerke et al. (2015) presented the results of the first $4 \mathrm{yr}$ of such a survey undertaken with the Large Binocular Telescope (LBT) and found one good failed SN candidate. This source, in NGC 6946 at RA 20:35:27.56 and Dec. +60:08:08.29, which we will hereafter refer to as N6946-BH1, experienced an outburst in 2009 March, first brightening to $\gtrsim 10^{6} \mathrm{~L} \odot$ but then fading to $\sim 10^{5} \mathrm{~L} \odot$ below its pre-outburst luminosity. Gerke et al. (2015) found that a coincident source experienced a similar, but slower outburst in the mid-IR. Gerke et al. (2015) also identified the progenitor in earlier archival Hubble Space Telescope (HST) images with 23.09 \pm 0.01 in $F 606 W$ and $20.77 \pm 0.01$ in $F 814 W$. Reynolds, Fraser $\&$ Gilmore (2015) performed a similar search for failed SNe using archival HST data and also identified a candidate.

A new kind of search is vulnerable to new kinds of false positives. The initial candidate selection was based on a decline in multiple optical bands, but a surviving star could be hidden by dust. There are several classes of sources known to have transient, heavily obscured phases. Some variable stars, such as R Cor Bor stars, may become optically faint for hundreds of days due to dust forming in their atmospheres (O'Keefe 1939). Stellar mergers can cause the envelope of the primary star to be ejected at low velocities - ideal conditions for dust formation - and result in a merger remnant that is luminous in the IR but optically obscured (Crause et al. 2003; Pejcha, Metzger \& Tomida 2016a,b). Luminous blue variables may experience eruptive mass-loss that obscures a surviving star following a weak transient - an SN 'impostor' - as $\eta$ Carinae did in the mid-1800s (e.g. Humphreys \& Davidson 1994; Smith et al. 2011b). There are also SN 'impostors' that arise from self-obscured superasymptotic giant branch (AGB) stars - SN 2008S-like transients - that quickly become re-enshrouded in dust (Prieto et al. 2008; Thompson et al. 2009; Kochanek 2011), though some SN 'impostors', from both super-AGB stars and more massive stars, may be lower luminosity SNe (see Adams \& Kochanek 2015; Adams et al. 2016a). Thus, multiwavelength follow-up is needed to vet failed SN candidates and determine whether the star survived.

In this work, we present follow-up observations and analysis of N6946-BH1. New HST imaging confirms that the identified progenitor has disappeared in the optical but a fainter, coincident source is detected in the near-IR (see Fig. 1). We present the data in Section 2 and describe our spectral energy distribution (SED) modelling in

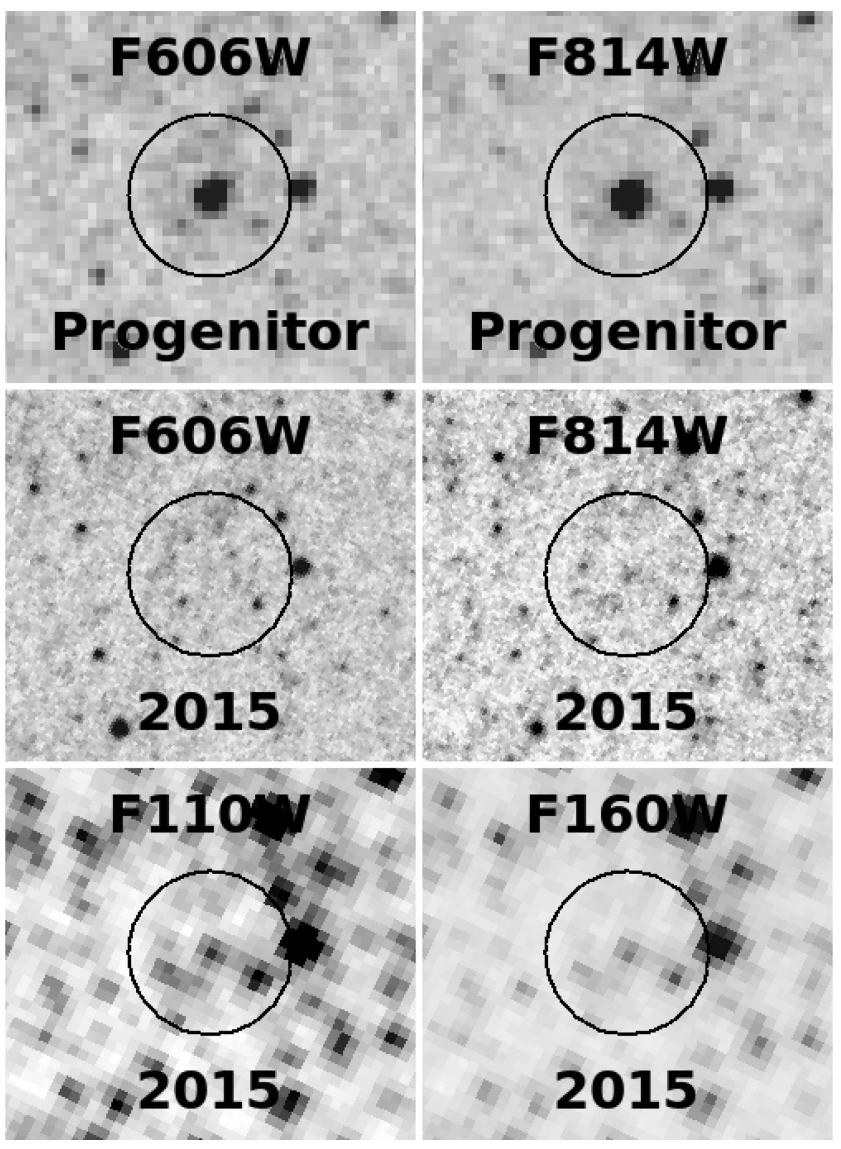

Figure 1. HST images of the region surrounding N6946-BH1. The top row shows the WFPC2 F606W (left) and $F 814 W$ (right) progenitor images. The middle row shows the corresponding 2015 WFC3 images and the bottom row shows WFC3/IR F110W (left) and F160W (right) images. The circles have a radius of 1 aresec. The progenitor has dramatically faded in the optical but there is still faint near-IR emission.

Section 3. In Section 4, we present detailed analysis of the progenitor, the outburst and the post-outburst observational constraints. While we find the failed SN interpretation for this event to be the most compelling, we consider alternative explanations in Section 5 before closing with our summary and conclusions in Section 6. Following Gerke et al. (2015), we adopt a distance of $5.96 \mathrm{Mpc}$ to NGC 6946 (Karachentsev, Sharina \& Huchtmeier 2000) and a Galactic foreground extinction of $E(B-V)=0.303$ based on the Schlafly $\&$ Finkbeiner (2011) recalibration of Schlegel, Finkbeiner \& Davis (1998). In this paper, all magnitudes are in the Vega system.

\section{DATA}

N6946-BH1 was discovered as part of our survey for failed SNe with the LBT (Kochanek et al. 2008; Gerke et al. 2015). The survey is still ongoing, and in this paper we utilize $38 U B V R$ epochs from the survey spanning from 2008 May 3 to 2016 December 8 (Adams et al. 2016b). We calibrated the LBT $U$-band photometry using Botticella et al. (2009), the $B$-band using SINGS ancillary data (Kennicutt et al. 2003) and the $V$ and $R$ bands from Welch et al. (2007). We performed image subtraction using ISIS (Alard \& Lupton 1998; Alard 2000) with reference images generated by combining the epochs in the top quintile of seeing. The $R$ and $V$ magnitude 'zero-points' are based on point spread function (PSF) photometry while $B$ and $U$ come from aperture photometry. All 
Table 1. Late-time variability constraints.

\begin{tabular}{lccc}
\hline Filter & $\begin{array}{c}\text { Variability } \\
\left(\mathrm{L}_{\odot} \mathrm{yr}^{-1}\right)\end{array}$ & Date range & $\begin{array}{c}\text { Number } \\
\text { of epochs }\end{array}$ \\
\hline$U$ & $-140 \pm 450$ & $2011-06-04-2015-12-07$ & 20 \\
$B$ & $-80 \pm 160$ & $2011-06-04-2015-05-20$ & 24 \\
$V$ & $-10 \pm 110$ & $2011-06-04-2015-10-13$ & 24 \\
$R$ & $-30 \pm 80$ & $2011-09-19-2015-12-07$ & 22 \\
\hline
\end{tabular}

photometry is presented in Table A1. We also use image subtraction to place constraints on the late-time variability of N6946-BH1. These constraints include an estimate of the systematic uncertainties based on the standard deviation of aperture fluxes measured on a grid of points within 10 arcsec of the progenitor location on the difference images after $3 \sigma$ clipping for each epoch. Since the brief optical spike in 2009 the optical fluxes have been consistent with no variability at the level of $\sim 200 \mathrm{~L}_{\odot} \mathrm{yr}^{-1}$, or roughly $10^{-3}$ of the progenitor flux (see Table 1 and Fig. 2).

Since the progenitor was only present in the LBT survey data for three epochs spanning less than 7 months prior to the start of its outburst, we also searched for archival data beyond the HST images identified in Gerke et al. (2015). We found the progenitor in two archival Canada-France-Hawaii Telescope (CFHT) MegaPipe images (Gwyn 2008). The transformed (to Johnson using the prescription in Gwyn 2008) MegaCAM apparent aperture magnitudes were $R=21.3 \pm 0.3, V=21.7 \pm 0.1, B=22.8 \pm 0.1$ and $U=23.5$ \pm 0.2 on 2005 July 2 and $R=21.5 \pm 0.3$ and $V=21.7 \pm 0.2$ on 2003 October 23.

There are also archival images of the progenitor from the Isaac Newton 2.5-m Telescope (INT). For these data we performed PSF photometry using DАОРНОт. Calibrating the $r$ - and $i$-band data with the Pan-STARRS PS1 catalogues (Chambers et al. 2016) and the $B$ - and $V$-band data by bootstrapping from LBT $B$ - and $V$-band photometry, we measure $B=23.3 \pm 0.1, r=22.0 \pm 0.1$ and $i=21.4 \pm 0.1$ on 1999 June $10 / 12$ and $V=22.3 \pm 0.1$ and $i=21.6$ \pm 0.1 on 2002 August 12 .

We supplement our coverage of the transient with public archival data from the Palomar Transient Factory (PTF) Data Release 2 (Law et al. 2009; Ofek et al. 2012; Laher et al. 2014). We adopt the adaptive aperture 'MAGAUTO' fluxes and upper limits from the PTF catalogues. The transient is detected in the first available epoch on 2009 March 17 at $R=19.0 \pm 0.1$ and is last detected at $R=19.6 \pm 0.1$ on 2009 May 26 .

We also use unfiltered images taken by amateur astronomer Ron Arbour between 2015 October 5 and 2016 January 23 to help constrain the peak magnitude and the start date of the optical transient. In these images, no source is detected at the position of N6946-BH1 to limiting magnitudes of 17.5-18.8.

We utilize both new and archival HST data. For our program, we obtained new Wide Field Camera 3 (WFC3) UVIS F606W and $F 814 \mathrm{~W}$ and IR $F 110 \mathrm{~W}$ and $F 160 \mathrm{~W}$ images on 2015 October 8. We also use the archival HST Wide Field and Planetary Camera 2 (WFPC2) F606W and F814W images taken on 2007 July 8 (PI: M. Meixner, GO-11229) that were considered in Gerke et al. (2015).

We aligned the new and archival HST images using the TWEAKREG and TWEAKBACK tasks in the DRIZZLEPAC package with rms errors in the astrometry of 0.04 arcsec for the UVIS images and 0.07 arcsec for the IR images. We calculated PSF magnitudes for the new HST data using the software package DоLРнот 2.0 (Dolphin 2000) ${ }^{1}$ with

\footnotetext{
${ }^{1} \mathrm{http}: / /$ americano.dolphinsim.com/dolphot/
}

the same parameter settings as in Adams \& Kochanek (2015). We used the drizzled HST WFPC2 $F 814 \mathrm{~W}$ pre-outburst image from 2007 as the reference in order to obtain PSF photometry at the progenitor location. The progenitor has clearly disappeared in the optical (see Fig. 1). The closest DOLPHOT source is 0.045 arcsec from the progenitor position, which is roughly consistent given the astrometric uncertainties. We also calculated a local World Coordinate System (WCS) alignment between the new and archival HST images using the IRAF task GEOMAP with rms errors in the astrometry of 0.012 arcsec for the UVIS images and 0.019 arcsec for the IR images. Using the new HST F814W $(F 110 W)$ as the reference for the local WCS alignment yielded similar photometry for the closest DOLPHOT source but now only $0.022 \operatorname{arcsec}(0.017 \operatorname{arcsec})$ from the progenitor location.

We estimate the likelihood that the $H S T$ source detected after the outburst is an incidental detection of an unrelated source based on the surface density of similarly bright sources. The surface density of all DOLPHOT sources within 4 arcsec of N6946-BH1 is $5.1 \mathrm{arcsec}^{-2}$, which corresponds to a 15 per cent chance of an unrelated source being detected within 0.045 arcsec (the distance of the closest latetime $H S T$ source from the progenitor location). For sources as bright as the detection (in $F 814 \mathrm{~W}$ ), this drops to a surface density of $1.4 \operatorname{arcsec}^{-2}$ and a 0.9 per cent likelihood.

We also use both new and archival Spitzer Space Telescope (SST) data. For our program, we obtained new 3.6 and $4.5 \mu \mathrm{m}$ images taken on 2016 January 21. We supplemented the IR light curve with available archival images (program IDs: 159, 3248, 10136, 11063, 20320, 20256, 30292, 30494, 40010, 40619, 70040, 80015, 80196, 90124, 10081, 11084, 12000; PIs: J. Andrews, M. Kasliwal, R. Kennicutt, C. Kochanek, R. Kotak, W.P. Meikle, M. Meixner, B. Sugerman). We performed aperture photometry on the SST images using a 2.4 arcsec aperture with a $2.4-4.8$ arcsec radius sky annulus and the standard aperture corrections from the Infrared $\mathrm{Ar}$ ray Camera (IRAC) instrument handbook. Since the SST was not designed to have the resolution for extragalactic stellar photometry, it is challenging to accurately measure the absolute photometry of N6946-BH1. We use IsIS to obtain an accurate measurement of the differential light curve, again estimating systematic uncertainties for each subtracted image from the standard deviation of fluxes on a grid of points within 15 arcsec of N6946-BH1 after $3 \sigma$ clipping. The light curve shows that the IR flux is rising from the first SST observations in 2004. Given the low surface density of variable sources, we attribute the change in IR flux coincident with N6946-BH1 to the source. The IR flux is significantly above its minimum value at all epochs that we model except for the first (2005-07-02) and last (2016-01-21). Thus it reasonable to treat the IR flux measurements as detections at every epoch except for those two. We estimate the odds that the remaining IR flux in 2016-01-21 could be the result of confusion. Using a grid of apertures within a 15 arcsec radius of N6946-BH1 we find that 7.7 percent and 1.8 percent of the apertures are brighter than our 3.6 and $4.5 \mu \mathrm{m}$ measurements, respectively.

In the latest epoch (2015 October 8 for HST and 2016 January 21 for $S S T$ ), it is plausible that only the near-IR photometry are detections of N6946-BH1. In addition to the IR flux being at or similar to their lowest measurements, DOLPHOT reports the 'sharpness' parameter for the $F 814 \mathrm{~W}$ emission in 2015 to be -0.48 , which suggests that the emission in this filter is not resolved, and the $S / N$ of the $F 606 \mathrm{~W}$ emission is only 2.4. Thus, we will consider both cases where all photometry in the latest epoch are detections and when all photometry except the near-IR measurements are taken as only upper limits. 


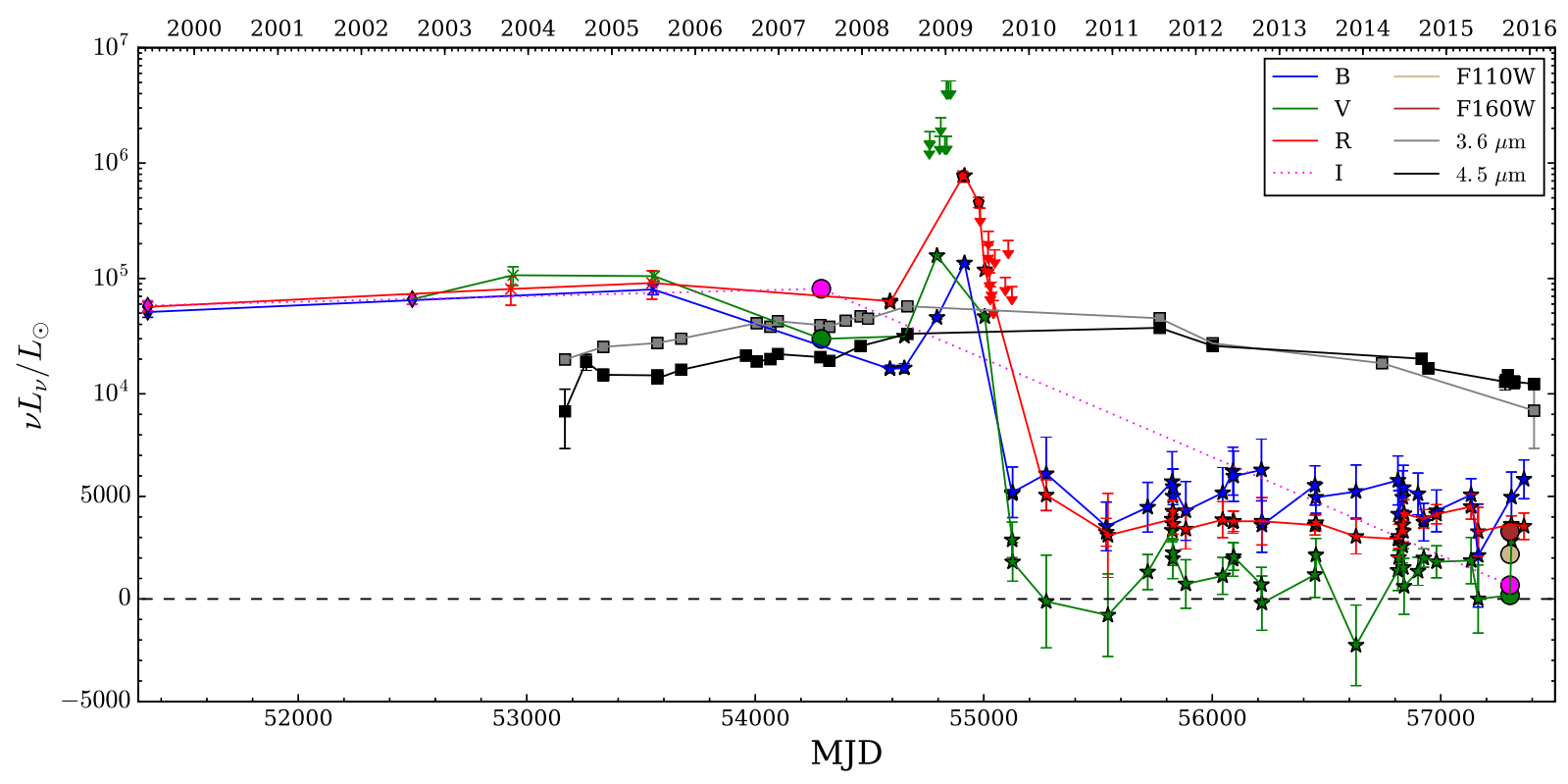

Figure 2. N6946-BH1 light curves from HST (large circles), SST (squares), LBT (stars), CFHT (x's), INT (diamonds), PTF (red pentagons and upper limits) and amateur astronomer Ron Arbour (green upper limits). The vertical axis switches from a linear scale below $10^{4} \mathrm{~L}_{\odot}$ to a logarithmic scale above $10^{4} \mathrm{~L}_{\odot}$. A luminosity of zero is given by the dashed black line for comparison. The uncertainties for the differential LBT and SST photometry do not include the significant uncertainty in their 'zero-points' created by crowding. The LBT fluxes at late times could easily be zero. For this purpose, the high-resolution HST constraints that any remaining optical flux is $<10^{3} \mathrm{~L}_{\odot}$ are more relevant.

\section{SED MODELLING}

We model the SED to constrain the physical properties (i.e. luminosity, temperature, mass and recent mass-loss) of the progenitor, its outburst and the late-time source. We will use the results of these models to discuss whether the progenitor survived the outburst and whether a failed $\mathrm{SN}$ or some other phenomenon (e.g. stellar merger or eruptive mass-loss) best explains the data.

Following the methods in Adams \& Kochanek (2015) and Adams et al. (2016a), we model the SED of the progenitor, its outburst and the late-time source using the dust radiative transfer code DUSTY (Ivezic \& Elitzur 1997; Ivezic, Nenkova \& Elitzur 1999; Elitzur \& Ivezić 2001). We use stellar models from Castelli \& Kurucz (2004) for stars with solar metallicity and effective temperatures between 3500 and $50000 \mathrm{~K}$ and revert to blackbody models when attempting to fit temperatures below $3500 \mathrm{~K}$. We employ a Markov Chain Monte Carlo (MCMC) wrapper around DUSTY to find best-fitting models and allowed parameter ranges. We adopt minimum photometric uncertainties of 10 per cent (to account for uncertainty in distance and metallicity and any systematic problems in the models). We use silicate dust from Draine \& Lee (1984) with a standard MRN grain size distribution $\left(\mathrm{d} n / \mathrm{d} a \propto a^{-3.5}\right.$ with $0.005<a<0.25 \mu \mathrm{m}$; Mathis, Rumpl \& Nordsieck 1977).

The IR variability of the progenitor and the post-outburst IR emission could be indicative of dust formation. We consider two modes of mass-loss: the ejection of a dusty shell and a steady-state wind. We assume that all dust formation occurs in the outflowing material once it cools to the dust formation temperature, $T_{\mathrm{f}} \simeq 1500 \mathrm{~K}$. In the shell model, as the shell continues to expand beyond the dust formation radius, $R_{\mathrm{f}}$, the optical depth, $\tau$, decreases, asymptoting at late times to $\tau \propto t^{-2}$, where $t$ is the elapsed time since the ejection of the shell. For a thin shell, the mass of the ejecta, $M_{\mathrm{ej}}$, corresponding to a given optical depth is

$M_{\mathrm{ej}}=\frac{4 \pi v_{\mathrm{ej}}^{2} t^{2} \tau_{V, \mathrm{tot}}(t)}{\kappa_{V}}$, where $v_{\text {ej }}$ is the velocity of the ejected shell and $\kappa_{V}$ is the opacity of the dust at $V$ band. As noted in Tables 2-4, for the shell models we generally fix the ratio between the inner and outer edges of the dust shell, $R_{\text {out }} / R_{\text {in }}$, to 2 . The models where we allow $R_{\text {out }} / R_{\text {in }}$ to vary show that the shell thickness is relatively unconstrained by the data and has little effect on estimates of the other model properties.

For a set of post-outburst shell models (labelled as 'with $\mathrm{d} L / \mathrm{d} t$ ' in Table 4) we also include constraints on the late-time variability of the source. As discussed in Adams \& Kochanek (2015), the luminosity of a surviving source of constant intrinsic luminosity is constrained by the variability, $\mathrm{d} L_{f, \text { obs }} / \mathrm{d} t$, and optical depth of the source in that filter, $f$, according to

$L_{*, f} \simeq \frac{1}{2} \frac{t}{\tau_{f, \text { eff }}}\left(\frac{\mathrm{d} L_{f, \text { obs }}}{\mathrm{d} t}\right) \mathrm{e}^{\tau_{f, \text { eff }}}$.

We impose the variability constraints, $\mathrm{d} L_{\mathrm{obs}} / \mathrm{d} t$, from Table 1 on the models by adding contributions of

$\chi_{f}^{2}=\left(\frac{\mathrm{d} L_{f, \text { obs }} / \mathrm{d} t-\mathrm{d} L_{f, \text { mod }} / \mathrm{d} t}{\sigma_{\mathrm{d} L_{f, \text { obs }}} / \mathrm{d} t}\right)^{2}$

for each constrained filter, $f$, where the model variability, $\mathrm{d} L_{f, \bmod } / \mathrm{d} t$, is

$\frac{\mathrm{d} L_{f, \bmod }}{\mathrm{d} t}=\frac{2 L_{f} \tau_{f, \mathrm{eff}}}{t}$

(see Adams et al. 2016a). We also consider a set of models where we compare the evolution of the IR flux to the expansion (and cooling) of the dust shell. For these models we compute the $\chi^{2}$ of a given MCMC step for the latest photometric constraints. We infer a shell expansion velocity, $v_{\mathrm{ej}}$, based on the elapsed time, $t$, and inner shell edge, $R_{\text {in }}$, of the model. We then extrapolate the model back to an earlier post-outburst epoch with SST observations using this $v_{\text {ej }}$ to find the appropriate $R_{\text {in }}$ for the earlier epoch, generating a new DUSTY model with the optical depth, $\tau$, expected from a $\tau \propto t^{-2}$ scaling, and include the $\chi^{2}$ for this extrapolated model in the MCMC step. 
Table 2. Progenitor SED models.

\begin{tabular}{lccccccccc}
\hline Model & Date & $\log L / \mathrm{L} \odot$ & $T_{*}(\mathrm{~K})$ & $\tau_{V, \text { tot }}$ & $T_{\mathrm{d}}(\mathrm{K})$ & $R_{\text {out }} / R_{\text {in }}$ & $\log M_{\text {ej }} / \mathrm{M}_{\odot}$ & $E(B-V)$ & $\chi_{\text {min }}^{2}$ \\
\hline P1 & $2005-07-02$ & $5.31_{-0.07}^{+0.12}$ & $6700_{-990}^{+1200}$ & $1.4_{-0.4}^{+0.4}$ & $1720_{-400}^{+240}$ & $2.5_{-1.3}^{+26.3}$ & $-6.8_{-0.1}^{+0.2}$ & $0.01_{-0.01}^{+0.14}$ & 1.5 \\
P2 & $2007-07-08$ & $5.30_{-0.05}^{+0.05}$ & $4310_{-380}^{+750}$ & $3.4_{-1.0}^{+0.9}$ & $1580_{-520}^{+360}$ & $7.9_{-6.5}^{+71.5}$ & $-6.5_{-0.2}^{+0.3}$ & $0.00_{-0.00}^{+0.05}$ & 0.5 \\
P3 & $2008-07-05$ & $5.35_{-0.07}^{+0.36}$ & $5580_{-2060}^{+850}$ & $3.9_{-1.9}^{+1.3}$ & $1550_{-140}^{+390}$ & $2.6_{-1.3}^{+67.0}$ & $-6.2_{-0.2}^{+2.8}$ & $0.01_{-0.01}^{+0.02}$ & 0.5 \\
P4 & $2005-07-02$ & $5.28_{-0.06}^{+0.07}$ & $6610_{-940}^{+1150}$ & $1.4_{-0.2}^{+0.2}$ & $1740_{-400}^{+220}$ & 2 (fixed) & $-4.6_{-0.2}^{+0.4}$ & 0 (fixed) & 2.1 \\
P5 & $2007-07-08$ & $5.29_{-0.06}^{+0.04}$ & $3260_{-320}^{+1670}$ & $1.5_{-1.4}^{+2.0}$ & $1300_{-1240}^{+590}$ & 2 (fixed) & $-4.3_{-1.3}^{+4.9}$ & 0 (fixed) & 0.8 \\
P6 & $2008-07-05$ & $5.55_{-0.06}^{+0.12}$ & $3590_{-80}^{+160}$ & $2.0_{-0.4}^{+0.5}$ & $940_{-530}^{+800}$ & 2 (fixed) & $-3.6_{-1.1}^{+1.4}$ & 0 (fixed) & 0.5 \\
P7 & $2007-07-08$ & $5.31_{-0.01}^{+1.5}$ & $3010_{-120}^{+6310}$ & 0 (fixed) & - & - & - & $0.02_{-0.01}^{+1.74}$ & 4.7 \\
P8 & $2005-07-02$ & $5.53_{-0.01}^{+0.01}$ & $4350_{-110}^{+100}$ & 0 (fixed) & - & - & - & 0 (fixed) & 33.2 \\
P9 & $2007-07-08$ & $5.30_{-0.01}^{+0.01}$ & $2960_{-90}^{+90}$ & 0 (fixed) & - & - & - & 0 (fixed) & 5.4 \\
P10 & $2008-07-05$ & $5.45_{-0.02}^{+0.02}$ & $2900_{-60}^{+60}$ & 0 (fixed) & - & - & - & 0 (fixed) & 15.7 \\
\hline
\end{tabular}

Notes. Constraints from MCMC models of the progenitor SED. The uncertainties give the 90 percent confidence intervals. $L_{*}$ is the bolometric luminosity of the source, $T_{*}$ is the intrinsic effective temperature of the input $\mathrm{SED}, T_{\mathrm{d}}$ is the dust temperature at the inner radius of the dust shell $\left(R_{\mathrm{in}}\right)$, $R_{\text {out }} / R_{\text {in }}$ is the thickness of the dust shell, $E(B-V)$ is the local $E(B-V)$ in NGC 6946 in addition to $E(B-V)=0.303$ adopted for Galactic extinction and $\chi^{2}$ is the fit of the model. For the $R_{\text {out }} / R_{\text {in }}=2$ models $M_{\text {ej }}$ is the ejected mass implied by equation (1), otherwise it is the mass-loss rate $\mathrm{M}_{\odot} \mathrm{yr}^{-1}$ implied by equation (5). Local extinction in NGC 6946 is not required to achieve good fits, but circumstellar dust is.

Table 3. SED outburst models.

\begin{tabular}{lcccccccc}
\hline Model & Date & $\log L / \mathrm{L}_{\odot}$ & $T_{*}(\mathrm{~K})$ & $\tau_{V, \text { tot }}$ & $T_{\mathrm{d}}(\mathrm{K})$ & $R_{\text {out }} / R_{\text {in }}$ & $\log M_{\mathrm{ej}} / \mathrm{M}_{\odot}$ & $\chi_{\text {min }}^{2}$ \\
\hline O1 & $2009-03-25$ & $6.33_{-0.09}^{+0.13}$ & $3300_{-210}^{+180}$ & 0 (fixed) & - & - & - & 0.9 \\
O2 & $2009-06-22$ & $5.47_{-0.12}^{+0.15}$ & $3190_{-220}^{+230}$ & 0 (fixed) & - & - & - & 5.1 \\
O3 & $2009-03-25$ & $6.51_{-0.26}^{+2.00}$ & $5080_{-1750}^{+27670}$ & $3.2_{-2.8}^{+4.3}$ & $280_{-230}^{+1180}$ & 2 (fixed) & $0.5_{-3.9}^{+5.3}$ & 0.0 \\
O4 & $2009-06-22$ & $6.54_{-1.11}^{+1.4}$ & $12010_{-8810}^{+30960}$ & $6.5_{-6.2}^{+1.5}$ & $390_{-330}^{+1450}$ & 2 (fixed) & $-0.1_{-4.2}^{+5.2}$ & 0.0 \\
O5 & $2009-03-25$ & $6.79_{-0.13}^{+0.14}$ & 3500 (fixed) & $2.0_{-0.7}^{+0.7}$ & $240_{-190}^{+1230}$ & 2 (fixed) & $-0.3_{-2.9}^{+3.7}$ & 2.5 \\
O6 & $2009-06-22$ & $5.94_{-0.13}^{+0.14}$ & 3500 (fixed) & $2.4_{-0.6}^{+0.6}$ & $310_{-260}^{+1240}$ & 2 (fixed) & $-1.5_{-2.5}^{+4.0}$ & 2.4 \\
\hline
\end{tabular}

Notes. Constraints from MCMC models of the SED during the outburst. The columns are the same as in Table 2, except the $E(B-V)$ is now fixed to the Galactic extinction for NGC 6946.

For the wind scenario, the inner edge of the dust is set by the formation radius $R_{\mathrm{f}}$ and we allow the thickness of the dust 'shell' to vary. Since the optical depth of a wind (or shell) is dominated by the inner edge, the results are usually insensitive to the thickness $R_{\text {out }} / R_{\text {in }}$. The mass-loss rate needed to produce a given optical depth is

$\dot{M}=\frac{4 \pi v_{\mathrm{w}} R_{\mathrm{f}} \tau_{V, \text { tot }}}{\kappa_{V}}$,

where $v_{\mathrm{w}}$ is the velocity of the wind.

\section{RESULTS}

We will, in turn, consider the implications of the SED constraints for the progenitor, the optical outburst and the post-outburst fate of the star. The models for these three phases are summarized in Tables 2-4.

\subsection{Progenitor}

As shown in Fig. 2, observations over the $10 \mathrm{yr}$ prior to the optical outburst reveal a very luminous $\left(10^{5.3} \mathrm{~L}_{\odot}\right)$ star that remained at a constant brightness between 1999 and 2005, but then faded in the optical between 2005 and 2008 as it brightened at $3.6 \mu \mathrm{m}$. We fit the SED for three progenitor epochs: summer 2005 with archival CFHT data (2005 July 2) and the nearest corresponding archival SST observations (2005 July 20), summer 2007 with archival HST (2007 July 8) and the nearest corresponding archival SST observa- tion (2007 July 3) and summer 2008 with the difference between the LBT pre-outburst images (2008 May 3-4 and 2008 July 5) and post-outburst images [ $\sim 25$ epochs between 2011 June 4 and 2015 December 7 together with archival SST observations from that summer (2008 July 7)]. ${ }^{2}$ The constraints from these fits are given in Table 2.

In Fig. 3 we show a colour-magnitude diagram of the bright stellar sources within a projected radius of 50 pc from N6946-BH1, corrected for Galactic extinction, along with evolutionary tracks for the PARSEC (v. 1.2S) stellar models (Bressan et al. 2012; Chen et al. 2015) of solar metallicity stars of various initial masses. The location of the theoretical main sequence relative to the location of the nearby stars in the colour-magnitude diagram constrains the extinction in the local environment of N6946-BH1 to be $E(B-V)$ $\lesssim 0.2 \mathrm{mag}$. This is consistent with the constraints from the MCMC SED modelling of the progenitor which give $E(B-V) \lesssim 0.15$ (see models P1-P3 in Table 2). Accordingly we adopt $E(B-V)=0$ for the local environment in all other models (unless noted otherwise).

Good fits to the progenitor data are only obtained when allowing for the presence of circumstellar dust (models P1-P6). We show the best-fitting progenitor model for summer 2007 (model P5) in Fig. 4. The low stellar temperatures and hot dust temperatures in the

\footnotetext{
${ }^{2}$ We use the difference since the effect of crowding in ground-based photometry is likely larger than the small flux remaining in the late-time HST imaging. However, we note that adopting instead the pre-outburst LBT constraints has little effect on the results.
} 
Table 4. Late-time SED models.

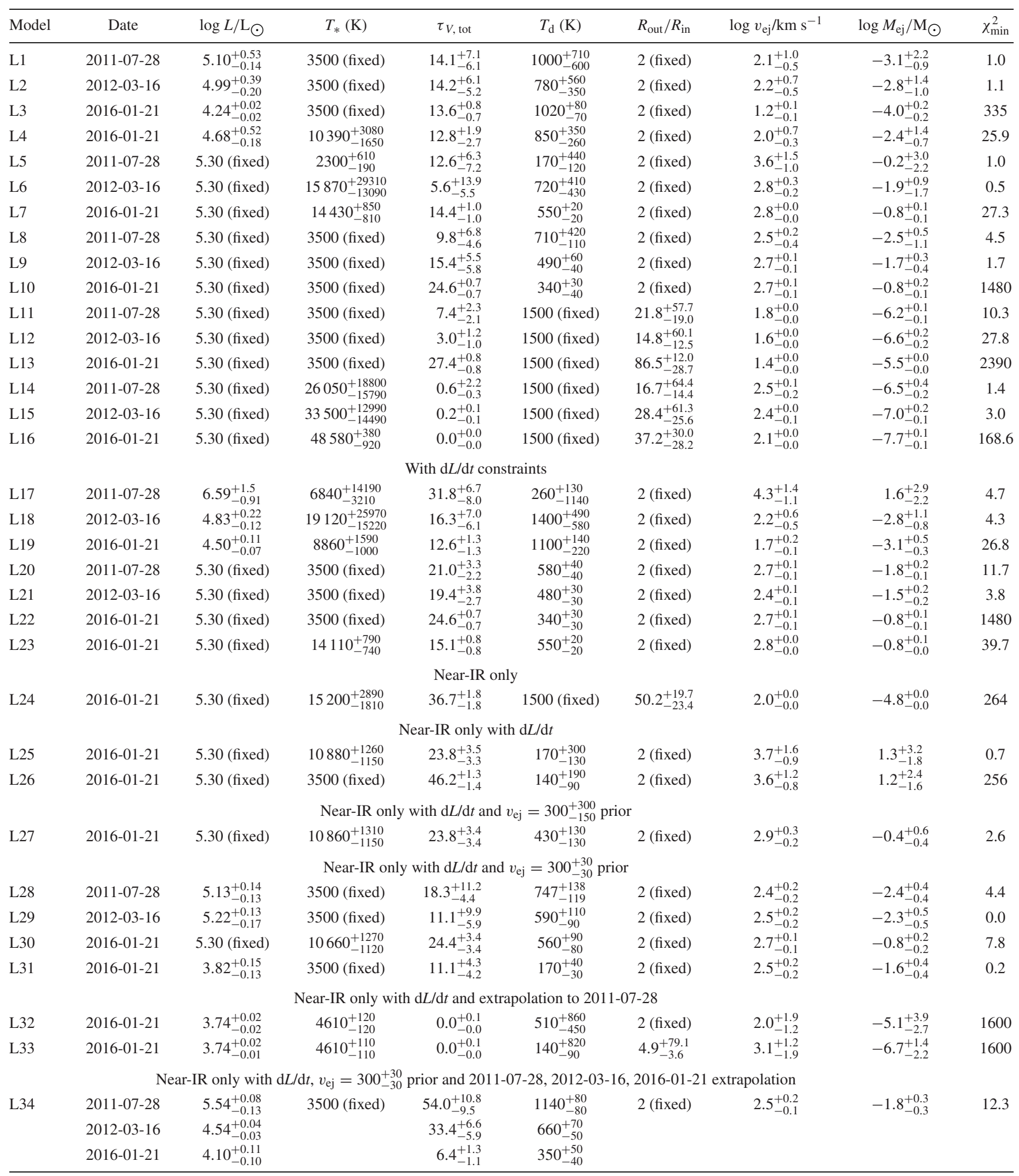

Notes. Constraints from MCMC models of the late-time SED. The columns are the same as in Table 3, but with the addition of $v_{\mathrm{ej}}$, the velocity required for material ejected on 2009 March 25 to reach the inner edge of the dust shell.

three progenitor epochs are consistent with an RSG emitting a dusty wind. The three epochs of progenitor fits hint that the progenitor may have had a decreasing effective temperature and a slightly increasing mass-loss rate, but given the uncertainties it is difficult to make a definitive statement. Between the 2007 and 2008 epochs, the IR luminosity increased more than the optical luminosity decreased, suggesting that the bolometric luminosity may have started to rise months before the observed optical transient. 


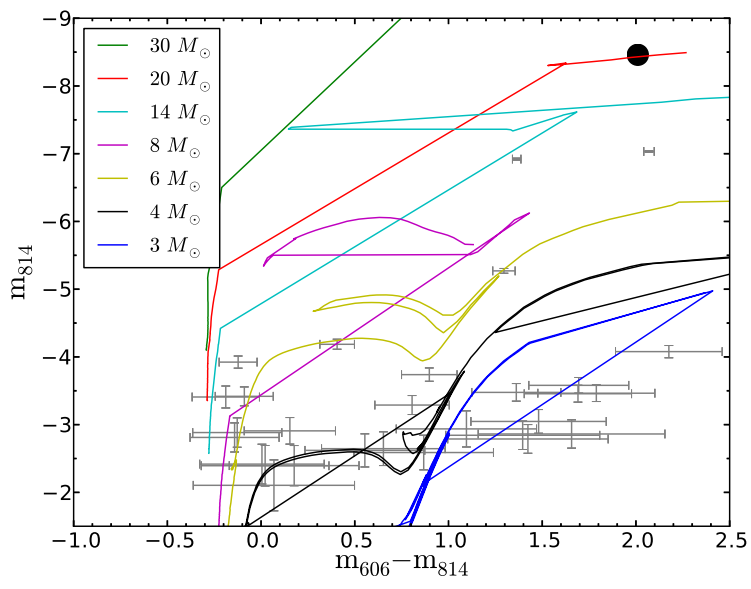

Figure 3. Colour (absolute) magnitude diagram of stars within 1.8 arcsec (50 pc projected) of N6946-BH1, corrected for Galactic extinction and assuming no additional extinction in the local environment of NGC 6946, is shown in grey. The progenitor is shown by the large black circle (with the uncertainties smaller than the size of the symbol). For comparison, evolutionary tracks for solar metallicity stars of various initial masses are shown as solid lines (Bressan et al. 2012; Chen et al. 2015).

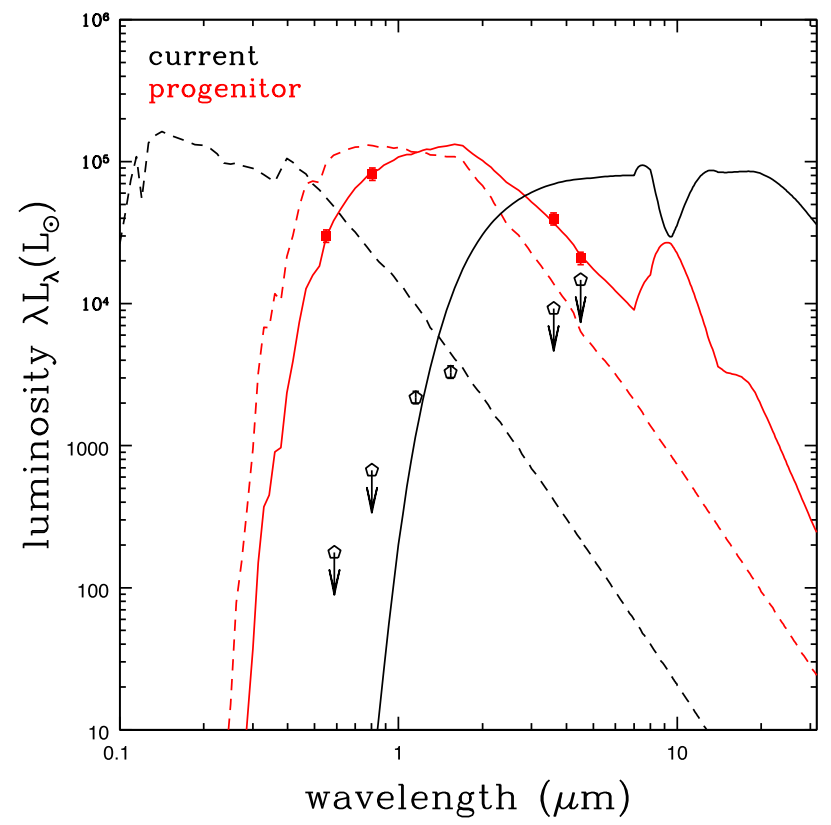

Figure 4. The SED of the progenitor of N6946-BH1 in 2007 (red) and of a possible surviving star in 2016 obscured by a dusty wind (black). The solid red line gives the best-fitting model (from P5 in Table 2) for the progenitor $\left(\log L_{*} / \mathrm{L}_{\odot}=5.29, T_{*}=4480 \mathrm{~K}, \tau_{V \text {, tot }}=3.3, T_{\mathrm{d}}=1800 \mathrm{~K}\right.$ and $\left.\chi^{2}=0.8\right)$ and the dashed red line gives the corresponding unobscured spectrum. The red squares give the HST and SST constraints in 2007. The open black pentagons give the latest $H S T$ and $S S T$ constraints on a surviving star when treating our $F 110 \mathrm{~W}$ and $F 160 \mathrm{~W}$ photometry as detections and the other bands as upper limits. The best-fitting model (from L25 in Table 4) for a surviving star with the luminosity of the progenitor $\left(\log L_{*} / \mathrm{L}_{\odot}=5.3\right)$ obscured by a dusty wind $\left(T_{\mathrm{f}}=1500 \mathrm{~K}\right)$ and shown by the solid black line has $T_{*}=14500, \tau_{V, \text { tot }}=37.3, R_{\text {out }} / R_{\text {in }}=72$ and a $\chi^{2}$ of 264 . Clearly, this 'best-fitting' model is in gross disagreement with the latest photometric constraints. A surviving star cannot be hidden by a dusty wind because the hot dust that dominates the obscuration reradiates the stellar emission in the near- to mid-IR. In order to hide the luminosity of the progenitor, the bulk of the emission must be radiated by cooler dust at wavelengths redward of $4.5 \mu \mathrm{m}$.

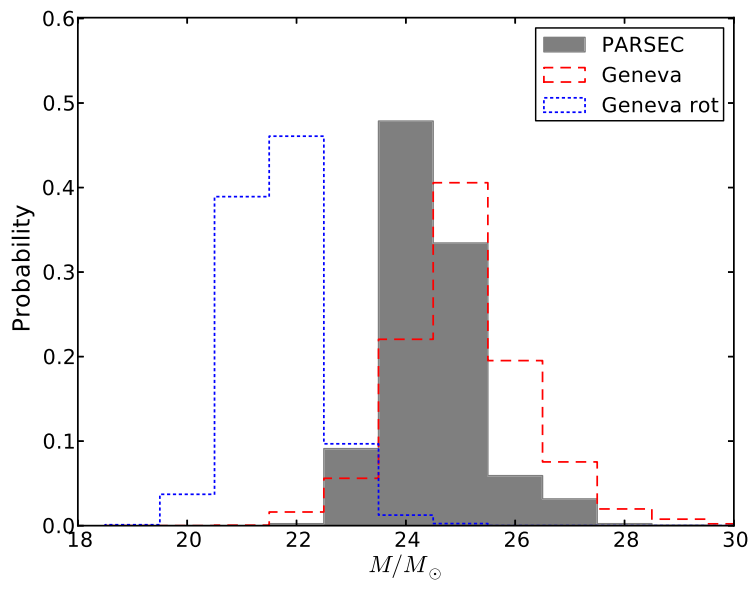

Figure 5. Progenitor mass probability distribution function based on MCMC fits to the pre-outburst HST and SST photometry (model P2) and the PARSEC (shaded grey) and Geneva rotating (blue dotted line) and non-rotating (red dashed line) stellar models when allowing $E(B-V)$ and $R_{\text {out }} / R_{\text {in }}$ to vary.

We matched the estimated progenitor luminosity and effective temperature estimates from the MCMC realizations with those from the PARSEC and Geneva stellar models (Ekström et al. 2012; Yusof et al. 2013). The PARSEC and the non-rotating Geneva models yield a probability distribution for the progenitor mass peaking at $\sim 25 \mathrm{M}_{\odot}$ while the distribution for the rotating Geneva models peaks at a slightly lower mass of $\sim 22 \mathrm{M}_{\odot}$ (see Fig. 5). This mass lies directly within the regime expected by the missing RSG problem (Kochanek et al. 2008; Smartt et al. 2009; Smartt 2015) and corresponds to a progenitor with a high 'compactness' parameter that is considered most likely to result in a failed SN (O'Connor \& Ott 2011; Ugliano et al. 2012; Horiuchi et al. 2014; Sukhbold \& Woosley 2014; Pejcha $\&$ Thompson 2015; Sukhbold et al. 2016).

\subsection{Outburst}

The outburst is not well sampled in time, but we can set useful constraints on the duration and minimum peak luminosity of the event. The source appeared quiescent in LBT observations on 2008 July 5 , but had brightened by a factor of several in observations taken on 2008 November 25. The peak observed (apparent) magnitudes occurred in the following LBT epoch on 2009 March 25 with $R=19.0, V=20.0$ and $B=22.1$, corresponding to luminosities $\left(v L_{v}\right)$ of $7.8 \times 10^{5}, 4.9 \times 10^{5}$ and $1.4 \times 10^{5} \mathrm{~L} \odot$ in $R, V$ and $B$, respectively (unfortunately there was no $U$-band data for this epoch). At the next LBT epoch on 2009 June 6, the transient had faded to $R=21.0, V=22.6, B=24.4$ and $U=24.7$, and by the subsequent LBT epoch on 2009 October 20 the source had already dropped below the progenitor flux in all optical bands (see Fig. 2).

PTF observations show that the source was present and likely already declining in flux from the earliest available images taken 2009 March 17. The transient is undetected in images from Arbour (private communication) with limiting magnitudes of $\sim 18.7$ in 2009 early January. Thus, the optical transient likely began between 2008 late November and 2009 mid-March and became optically faint between 2009 early June and 2009 late October, constraining the duration of the optical transient to be between 3 and 11 months. Unfortunately, there are no SST observations during the optical outburst, but it seems clear that the transient evolved more slowly in the IR than in the optical. 
We generated models of the SED for the two LBT epochs taken during the optical outburst (see Table 3). Without IR constraints there is a significant degeneracy, with hotter intrinsic temperatures yielding larger bolometric luminosities obscured by larger optical depths. Thus, we also present models with $T_{*} \equiv 3500$ since, in addition to being the likely temperature of the progenitor, the colour temperatures for the failed SN transients powered by hydrogen recombination in the models of Lovegrove \& Woosley (2013) are relatively cool. The peak luminosity was at least $10^{6} \mathrm{~L}_{\odot}$ (see model O1 in Table 3). The bolometric luminosity returned to roughly that of the progenitor by the first SST epoch after the peak of the optical outburst, which, unfortunately, was not taken until 2011 July 27.

We can also use the minimum and maximum durations of the optical transient to set constraints on the velocity of material ejected during the event if we assume that the optical flux collapses before the IR flux due to the formation of dust in the newly ejected material. This seems a reasonable assumption given that all models of the SED after the optical disappearance of the source (except for shell models for 2012-03-16 and 2011-07-28 that allow very hot stellar temperatures) require much higher optical depths than models of the progenitor in order to produce good fits.

Given the SED modelling constraints on the source luminosity and temperature on 2011-07-28 and $T_{\mathrm{f}}=1500 \mathrm{~K}\left(L_{*}, T_{*}\right.$ and $T_{\mathrm{d}}$ set $R_{\mathrm{f}}$ ), material ejected at the start of the transient must have $170<v_{\text {ej }}<560 \mathrm{~km} \mathrm{~s}^{-1}$ in order to reach $R_{\mathrm{f}}$ and form dust to extinguish the optical flux within the time window of the observational constraints. A moderately hotter dust condensation temperature of $T_{\mathrm{f}}=2000$ would decrease the minimum velocity to $v_{\text {ej }} \simeq 75 \mathrm{~km} \mathrm{~s}^{-1}$.

These constraints on the peak luminosity, possible duration and ejected velocities are broadly consistent with the cool $(\sim 3000 \mathrm{~K})$, $\sim 1 \mathrm{yr}, \sim 10^{6} \mathrm{~L} \odot$ transient with $v_{\mathrm{ej}} \sim 100 \mathrm{~km} \mathrm{~s}^{-1}$ predicted by Lovegrove \& Woosley (2013) for a failed SN in an RSG unbinding the hydrogen envelope. Of course, with the very limited observations, the outburst may also be consistent with other types of events, such as an SN impostor or a stellar merger. Thus, we must rely on the late-time evolution of the source to decipher its true nature.

\subsection{Late time}

The LBT difference imaging shows that the optical flux collapsed by 2009 October 20 and has remained far below the flux of the progenitor since then (see Fig. 2). This result is confirmed by the late-time HST data from 2015 October 8 showing that the progenitor has clearly vanished in the optical, where the emission decreased by at least 5 mag (see Figs 1, 4 and 6). The mid-IR emission has evolved much more slowly than the optical, but the source is now fainter than the progenitor at $3.6 \mu \mathrm{m}$ and nearly as faint at $4.5 \mu \mathrm{m}$. There is still faint near-IR emission coincident with the progenitor location (unfortunately there is no progenitor near-IR data). We will present SED models for three late-time epochs for which there are both 3.6 and $4.5 \mu \mathrm{m}$ observations: 2011 July 28, 2012 March 16 and 2016 January 21 (see Table 4). We compliment these SST constraints with optical $V$ - and $R$-band constraints inferred from the HST (F606W and $F 814 W$ ) photometry on 2015 October 8 extrapolated to these three dates using the LBT constraints on the variability of the $V$ and $R$ band between 2011 and 2015 listed in Table 1 .

First, we consider whether the progenitor could have survived as a heavily obscured star with a steady-state wind or an ejected shell, given the late-time photometric constraints.

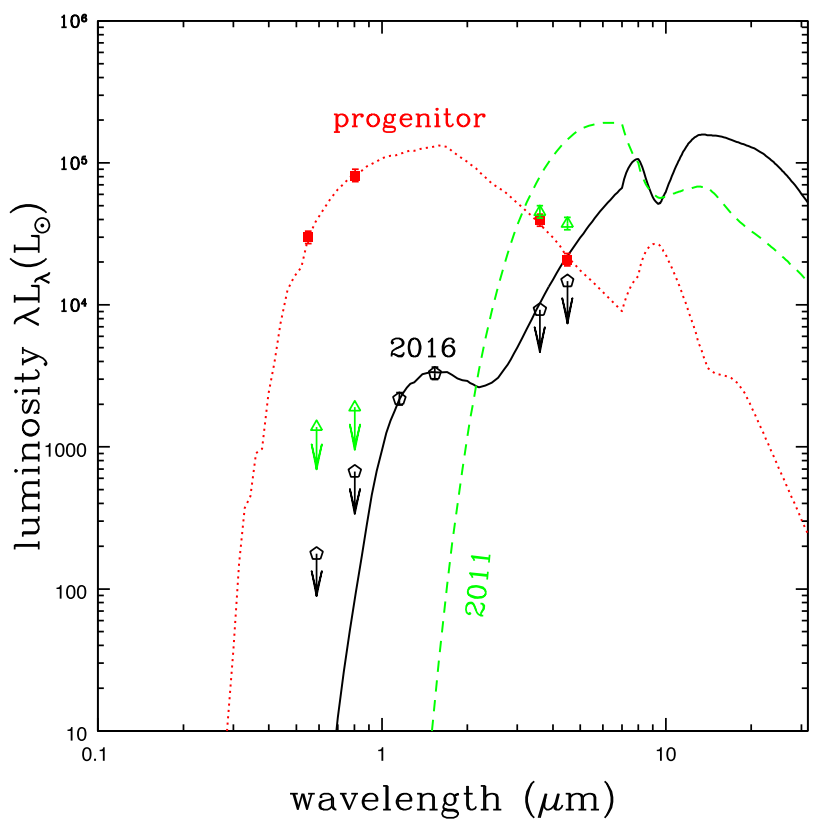

Figure 6. The SED of N6946-BH1 in 2016 with the best-fitting model (from L30 in Table 4) for a surviving star with the luminosity of the progenitor $\left(\log L_{*} / \mathrm{L}_{\odot}=5.3\right)$ obscured by a dusty shell when treating our $F 110 \mathrm{~W}$ and $F 160 \mathrm{~W}$ photometry as detections and the other bands as upper limits is given by the solid black line. The open black pentagons are the latest SED constraints from HST and SST. This particular model has $T_{*}=10700 \mathrm{~K}$, $\tau_{V, \text { tot }}=24.2, T_{\mathrm{d}}=570 \mathrm{~K}$ and $\chi^{2}=7.8$. The dashed green line gives the corresponding shell model for 2011 July 28 based on the implied $v_{\mathrm{e}}$ ( $\tau_{V \text {, tot }}=206$ and $T_{\mathrm{d}}=1400 \mathrm{~K}$ ) and the green triangles give the $S S T$ and LBT constraints for that epoch. The high optical depth required by the 2016 models correspond to such high optical depths in 2011 that the models are optically thick even at $3.6 \mu \mathrm{m}$, leading to a step drop in the SED blueward of $4.5 \mu \mathrm{m}$ that is in gross contradiction with the observational constraints. For comparison, the progenitor constraints and best-fitting model are given by the red squares and dotted line.

Models with the progenitor surviving behind a dusty wind do not fit the data well even if the surviving star's temperature is allowed to vary and all of the photometry is treated as upper limits except for the clear HST detections in the near-IR (see model L24 in Table 4 and Fig. 4). The basic issue is that even at high optical depths the models are unable to reradiate the progenitor's luminosity primarily at wavelengths redward of $4.5 \mu \mathrm{m}$, as required by the photometric constraints, because the bulk of the light is reprocessed by hot dust that produces flux in the near-IR (see Fig. 4). The $4.5 \mu \mathrm{m}$ constraint can only be avoided if the characteristic dust temperature is $\lesssim 600 \mathrm{~K}$, far below typical dust formation temperatures (see e.g. Gail \& Sedlmayr 2014). Thus, the progenitor did not survive behind a thick, dusty wind.

Models with the progenitor surviving behind an ejected shell are also inconsistent with the observational constraints. A $T_{*} \sim 3500 \mathrm{~K}$ progenitor hidden behind dust cannot easily reproduce the small slope between the near-IR constraints. A much hotter surviving star ( $T_{*} \sim 14000 \mathrm{~K}$ ) obscured by a dusty shell (model L23) is better able to match the near-IR constraints, although still with a best $\chi^{2}$ of 40 when including the variability constraints. Good fits can only be achieved by (in addition to allowing $T_{*}$ to vary) treating all of the photometry as upper limits except for the near-IR. In this case, a model with a hot star $\left(T_{*} \sim 11000 \mathrm{~K}\right)$ and a high optical depth $(\tau \sim 24)$ from cool dust $\left(T_{\mathrm{d}} \sim 170 \mathrm{~K}\right)$ can fit the data with $\chi^{2}$ $\simeq 0.7$ (see L25 in Table 4). However, such a cool dust temperature 


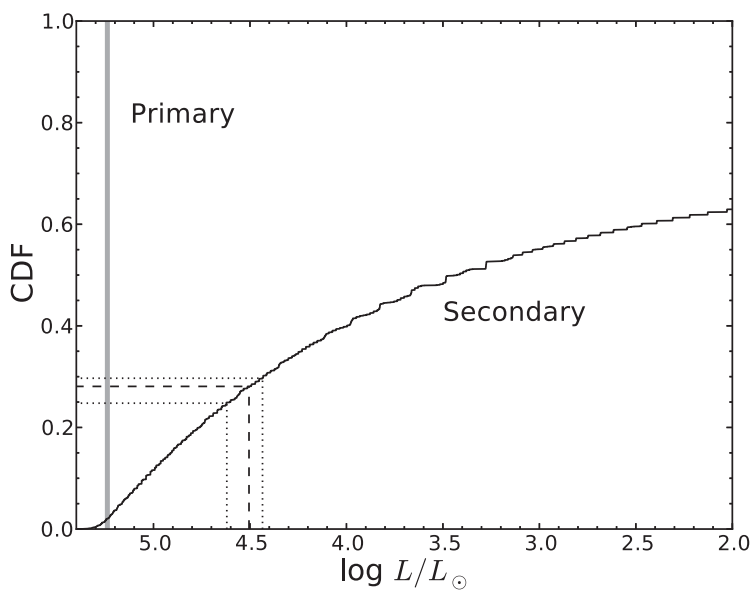

Figure 7. Cumulative distribution function for the luminosity of a surviving binary companion given our constraints on the progenitor mass (see Fig. 5). The dashed line indicates the median luminosity from the MCMC SED modelling of the late-time source and the corresponding likelihood of a surviving secondary being this luminous. Similarly, the dotted lines indicate the 10th and 90th percentile luminosities from the SED modelling. The thin vertical band shows the constraint on the progenitor luminosity.

requires a much higher ejecta velocity $\left(\sim 5000 \mathrm{~km} \mathrm{~s}^{-1}\right)$ than allowed by the constraints on the elapsed time between the start of the optical outburst and the collapse of the optical flux due to dust formation $\left(170<v_{\text {ej }}<560 \mathrm{~km} \mathrm{~s}^{-1}\right.$; see Section 4.2). Enforcing a prior on the ejecta velocity (with a 10 percent uncertainty) increases the dust temperature and also worsens the fit to $\chi^{2} \sim 7.8$ (see L30 in Table 4 and Fig. 6).

Another consideration is the evolution of the IR flux. The latetime $3.6 \mu \mathrm{m}$ flux decreases faster than the $4.5 \mu \mathrm{m}$ flux. Although this would be a natural consequence of the dust temperature decreasing as the ejected shell expands, the corresponding evolution of the optical depth is problematic for the models. The high optical depths required to fit the 2016 photometric constraints would correspond to high enough optical depths in 2011 to be optically thick even into the mid-IR, resulting in an SED in 2011 that would drop steeply blueward of $4.5 \mu \mathrm{m}$, in gross disagreement with the SST photometry from 2011 (see L32 in Table 4 and Fig. 6).

The conclusion we draw from this section is that the evolution of the SED can only be well fit by our models if the bolometric luminosity fades to be significantly below that of the progenitor. The high optical depth required by the late-time photometric constraints makes the result essentially independent of the stellar temperature or the details of the atmospheric models. What then would explain the late-time flux?

First, we consider whether the late-time emission could be due to a surviving binary companion obscured by dusty ejecta from the failed SN of the progenitor. Massive stars have a large multiplicity fraction (>82 per cent; Chini et al. 2012; Sana et al. 2012). We estimated the luminosity distribution of surviving companions of core-collapse $\mathrm{SNe}$ assuming passively evolving (i.e. no interactions) binaries with the progenitor masses drawn from the distribution in Fig. 5. We assumed a binary fraction of unity and a uniform secondary mass distribution following the simple models of Kochanek (2009). The secondary luminosity was the luminosity for a star with the mass of the secondary on the PARSEC isochrone corresponding to the lifetime of the primary. This yields the cumulative probability function of a surviving secondary brighter than a given luminosity shown in Fig. 7. If the binary fraction is unity, then there is a like- lihood of roughly 30 percent that the progenitor had a secondary at least as bright as the best estimate of $\log L / \mathrm{L}_{\odot}=4.50_{-0.07}^{+0.10}$ for the remaining luminosity in 2016 January (model L19; 90 per cent confidence intervals).

The likelihood drops in proportion to any reduction in the binary fraction. However, a surviving secondary would not explain the continued late-time decay of the bolometric luminosity implied by the mid-IR decay. The envelope of the secondary could have been shock heated by ejecta from the primary, making it overluminous, but the luminosity would only decay on a thermal time-scale ( $>10^{3} \mathrm{yr}$; Pan, Ricker \& Taam 2013; Shappee, Kochanek \& Stanek 2013).

The late-time emission could be powered by fallback accretion on to a newly formed black hole. Although fallback is most commonly discussed as a possible power source for gamma-ray bursts and other short-duration transients (Woosley 1993; MacFadyen \& Woosley 1999; Kashiyama \& Quataert 2015), fallback accretion may emit significant luminosity on much longer time-scales $(>\mathrm{yr})$ if the progenitor had sufficient angular momentum for material to circularize beyond the newly formed black hole's innermost stable circular orbit. Theoretical models have not been carried out for fallback accretion in the Lovegrove \& Woosley (2013) failed SN scenario, but Perna et al. (2014) find that for SN explosion energies leading to black hole remnants there is always a region of parameter space that leads to long-lived accretion discs. Given sufficient angular momentum, super-Eddington accretion may be maintained for $\sim 10$ yr with the accretion rate, $\dot{M}_{\mathrm{d}}$, predicted to be roughly $\propto t^{-4 / 3}$ (Perna et al. 2014). After the accretion becomes sub-Eddington, the accretion rate declines more slowly with $\dot{M}_{\mathrm{d}} \propto t^{-19 / 16}$ (Cannizzo, Lee \& Goodman 1990).

The evolution of the bolometric luminosity of N6946-BH1 may be well fit by a power law with a slope similar to these expectations for late-time fallback discs. For example, the inferred luminosity evolution implied by models O5, O6 and L34 for a transient constrained to have begun between 2008 November and 2009 March is $L \propto t^{-1.4 \pm 0.5}$ (see Fig. 8). The ejecta from failed $\mathrm{SNe}$, with their low velocities and relatively high densities, should be very efficient at forming dust (Kochanek 2014a). Dust in marginally bound or slowly ejected material could absorb the optical (and UV) flux emanating from a hot accretion disc and reradiate it at the longer wavelengths shown to be slowly fading by the SST data. Although we are adopting a stellar spectrum as an input to our SED models, the details of the temperature and spectral characteristics of the intrinsic spectrum are unimportant when the optical depth is high. We do, however, caution that the inferred slope for the bolometric evolution is model dependent and less robust than the conclusion that by 2016 the bolometric luminosity had faded to significantly below that of the progenitor.

\section{ALTERNATIVES}

We next consider alternative mechanisms for making a luminous star appear to vanish. Could the failed SN candidate be an exotic type of variable star? The optical luminosities of Mira variables can decline by up to 8 mag over periods of hundreds of days (Merrill 1940). As these pulsating stars cool, molecules such as titanium oxide can form high in the stellar atmosphere, reradiating the optical emission in the near-IR (Reid \& Goldston 2002). The optical diminution of Miras is not preceded by an outburst, like that of N6946-BH1. Moreover, N6946-BH1 has been optically faint for $7 \mathrm{yr}-$ much longer than the periods of Miras - and Miras do not experience changes in their bolometric luminosities as large as observed for N6946-BH1. 


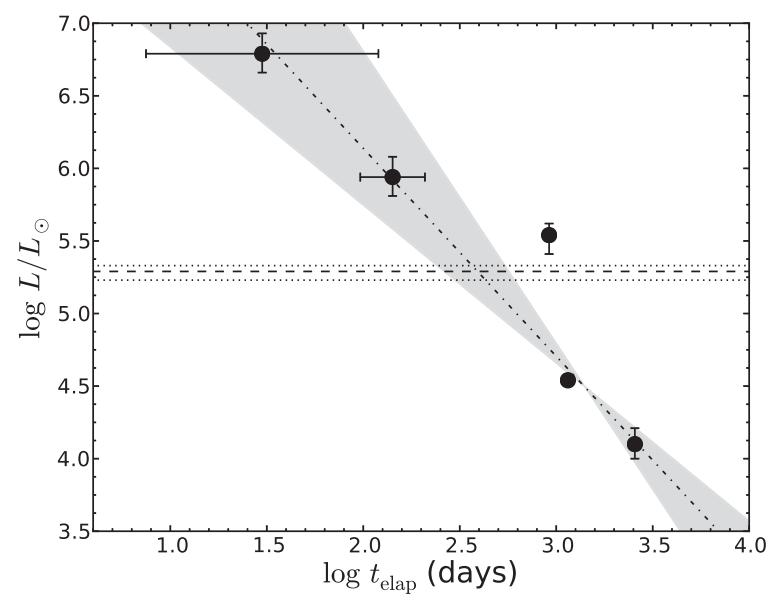

Figure 8. Evolution of the bolometric luminosity as a function of elapsed time since the start of the optical outburst (between 2008 November 25 and 2009 March 17) as inferred from the SED model. The vertical error bars on the points represent the 90 percent confidence intervals from the MCMC modelling assuming $T_{*}=3500 \mathrm{~K}$ (models $\mathrm{O} 5$ and $\mathrm{O6}$ ) and that at late times a dusty shell is expanding self-consistently with the inferred velocity constraints (model L34). The shaded grey region is defined by the bounds on the slope of the best-fitting linear regressions for the luminosity given the uncertainty in the start of the transient. The dot-dashed line has the slope of $-4 / 3$ expected for late-time fallback accretion discs (Perna et al. 2014). For reference, the horizontal dashed and dotted lines give the progenitor luminosity (on 2007-07-08 assuming $E(B-V)=0$; see P5 in Table 2) and its 90 per cent confidence interval.

$\mathrm{R}$ Coronae Borealis (R Cor Bor) stars are a rare class of cool, carbon-rich, hydrogen-poor supergiants that repeatedly, but irregularly, decline for up to 8 mag for hundreds to thousands of days, due to dust forming in the atmosphere (O'Keefe 1939; Clayton 2012). The absolute magnitudes of R Cor Bor stars range from $M_{V} \sim-5.2$ to $\sim-3.4$ at maximum (Tisserand et al. 2009), significantly fainter than the progenitor of N6946-BH1 $\left(M_{V} \simeq-6.8\right)$. Since R Cor Bor stars are thought to arise from a very late final shell flash in AGB stars or the merger of two white dwarfs (Webbink 1984; Iben, Tutukov \& Yungelson 1996; Clayton 2012) there is no reason to suspect that a similar phenomenon occurs at the higher luminosity of N6946-BH1, and R Cor Bor stars do not experience optical outbursts like that seen for N6946-BH1 prior to its disappearance.

An eclipsing binary with a long period, such as the recent one presented by Rodriguez et al. (2016), could possibly mimic the optical disappearance of N6946-BH1, but would not explain the optical outburst or the more gradual evolution of the IR luminosity.

Could N6946-BH1 instead be an SN 'impostor'? At $M_{R} \lesssim$ -10.64 , the peak luminosity of the outburst likely lies within the faint end of events frequently considered to be non-terminal outbursts of luminous blue variables (Smith et al. 2011b). The constraints on the ejecta velocity and transient duration are also consistent with the broad diversity of SN impostor properties. The driving mechanism behind eruptive mass ejections in these massive stars is unclear. There is significant evidence that some of these events may, in fact, be low-energy SNe (Adams \& Kochanek 2015; Adams et al. 2016a). The main evidence disfavouring N6946-BH1 being an SN 'impostor' is that after eruptive mass-loss a star is expected to be overluminous, not subluminous (Kashi, Davidson \& Humphreys 2016). The conclusion from Section 4.3 is that N6946$\mathrm{BH} 1$ has faded below the luminosity of its progenitor, which makes it unlikely that the star survived.
Although we only consider the simple case of obscuration of spherically symmetric dust, we expect this basic conclusion to largely be valid for shells with inhomogeneities and some asymmetries (see discussion in Kochanek, Szczygieł \& Stanek 2012). For example, Kashi \& Soker (2017) propose a complex dust geometry to evade the conclusions of our models with a spherical dust distribution. In essence, they propose a toroidal dust distribution viewed edge with the energy escaping along the axis of the torus without significant heating the torus. We used DUSTY to explore the limiting case of this geometry, a slab of dust illuminated from one side and observed from the other, and found that significant fractions of the energy were still transmitted through the slab and visible as hot dust emission even when the visual opacity of the slab was very large ( 25 per cent of the energy for $\tau_{V}=100$ ). In an actual torus, this fraction will increase because energy from heating the inner edge of the torus which is not transmitted will also contribute to the heating of other areas of the torus instead of simply escaping. In the vertical direction, the torus also has to have negligible optical depth because for a low optical depth the star will be visible in scattered light and for higher optical depth the geometry returns to being effectively spherical.

Our primary concern with the failed $\mathrm{SN}$ interpretation is that it does not provide a natural explanation for the rising IR flux in the final years prior to the optical outburst. Various mechanisms have been proposed to enhance mass-loss in the final years before an SN (e.g. Mcley \& Soker 2014; Shiode \& Quataert 2014), though mainly in the context of explaining precursor eruptions. Dramatic eruptions must be relatively rare $(\sim 10$ per cent $)$ given the relatively low frequency of Type IIn SNe. Even if precursor variability is a continuum, significantly enhanced mass-loss is likely not a generic outcome given that in the few cases where there are multi-epoch observations of the SN progenitor little, if any, variability is observed (see Kochanek et al. 2017).

A stellar merger could explain many of the properties of N6946$\mathrm{BH} 1$, as Roche lobe overflow could give rise to increasing IR and decreasing optical flux prior to an optical outburst triggered by contact (Pejcha 2014; Pejcha et al. 2016b; MacLeod et al. 2017). The subsequent inspiral of the secondary through the envelope could lead to significant mass-loss and dust formation (MacLeod et al. 2017). However, in such a scenario the mass outflow would likely be on order the escape velocity of the primary (Ivanova et al. 2013; Pejcha et al. 2016a), which for the RSG progenitor of N6946-BH1 would be somewhat lower than the velocity constraint we found in Section 4.2 (85-115 versus $170-560 \mathrm{~km} \mathrm{~s}^{-1}$ ). Also, the merger remnant would be expected to be significantly more luminous than the progenitor for $\sim$ a thermal time (Ivanova et al. 2013), as appears to be the case for the well-studied Galactic stellar mergers V838 Mon (Tylenda 2005), V4332 Sgr (Tylenda et al. 2005) and V1309 Scorpii (Tylenda \& Kamiński 2016), as well as for the recent proposed massive star mergers in NGC 4490 (Smith et al. 2016) and M101 (Blagorodnova et al. 2017).

\section{SUMMARY AND CONCLUSIONS}

We present new late-time $H S T, S S T$ and LBT observations of the failed SN candidate found by Gerke et al. (2015). We also analyse archival imaging of the progenitor and find that between 1999 and 2009 the progenitor maintained a roughly constant bolometric luminosity while fading in the optical and brightening in the mid-IR. The progenitor SED can be well fit by a $\sim 25 \mathrm{M}_{\odot}$ RSG. This lies directly within the mass range of the missing RSG SN progenitors and high core 'compactness' identified in theoretical models as most likely 
to give rise to failed SNe and direct black hole formation. In 2009 the progenitor underwent a weak $\left(\sim\right.$ few $\left.10^{6} \mathrm{~L}_{\odot}\right)$, but long $(3-11$ months), optical outburst. The transient is broadly consistent with the failed SN models of Lovegrove \& Woosley (2013), although the data on the optical transient are limited. Between 3 and 11 months from the start of the optical outburst the optical flux collapsed to be far below that of the progenitor, though the source faded more slowly in the mid-IR. This suggests that dust formed in material ejected during the outburst with $170 \gtrsim v_{\text {ej }}<560 \mathrm{~km} \mathrm{~s}^{-1}$. These constraints on the peak luminosity, transient duration and ejecta velocity are consistent with numerical simulations of failed $\mathrm{SNe}$ (Lovegrove \& Woosley 2013), but they also may be consistent with other phenomenon such an $\mathrm{SN}$ impostor or stellar merger.

Our late-time data show that the source is now $>5$ mag fainter than the progenitor in the optical and, though still fading, has returned to the pre-transient flux in the mid-IR. The bolometric luminosity of the source is fading with $L \propto t^{-1.4 \pm 0.5}$, which is consistent with the models of late-time fallback accretion on to a black hole (Perna et al. 2014). The bolometric luminosity of N6946-BH1 is now significantly fainter than the progenitor, suggesting that the progenitor did not survive. We propose that the late-time detection of near-IR emission may be due to fallback accretion on to a newly formed black hole obscured by dust that formed in a weakly ejected envelope.

N6946-BH1 merits further study. It would be valuable to constrain the luminosity variability of the progenitor at even earlier times with other archival data. New observations are also needed to confirm that N6946-BH1 is a failed SN. If the late-time luminosity is powered by fallback accretion, it may be possible to detect X-rays with the Chandra X-ray Observatory as long as the neutral hydrogen column depth is not too large. If X-rays are not detected it will be especially important to continue to monitor N6946-BH1 in the optical to make sure that the source does not start to rebrighten due to a decreasing optical depth and in the mid-IR to see if the $3.6 \mu \mathrm{m}$ and $4.5 \mathrm{~m}$ fluxes continue to decrease. Ultimately, observations at 10-20 $\mu \mathrm{m}$ with the James Webb Space Telescope may be needed to verify that a surviving star is not hidden by cooler dust than can be probed with SST. If confirmed, N6946-BH1 would be the first failed $\mathrm{SN}$ and first black hole birth ever discovered and would resolve the problem of the missing high-mass $\mathrm{SN}$ progenitors.

\section{ACKNOWLEDGEMENTS}

We thank Ron Arbour for sharing his images of NGC 6946. We thank Klaas Wiersema and Andrew Levan for identifying observations covering N6946-BH1 in the INT archive. We thank John Beacom and the anonymous referee for helpful comments on the manuscript. Financial support for this work was provided by the NSF through grant AST-1515876. This work is based in part on observations made with the Spitzer Space Telescope, which is operated by the Jet Propulsion Laboratory, California Institute of Technology under a contract with NASA, and in part on observations made with the NASA/ESA Hubble Space Telescope obtained at the Space Telescope Science Institute, which is operated by the Association of Universities for Research in Astronomy, Inc., under NASA contract NAS 5-26555. These observations are associated with program GO-14266. This work is based in part on observations made with the Large Binocular Telescope (LBT). The LBT is an international collaboration among institutions in the USA, Italy and Germany. The LBT Corporation partners are: The University of Arizona on behalf of the Arizona University System; Istituto Nazionale di Astrofisica, Italy; LBT Beteiligungsgesellschaft, Germany, represent- ing the Max Planck Society, the Astrophysical Institute Potsdam and Heidelberg University; The Ohio State University; The Research Corporation, on behalf of The University of Notre Dame, University of Minnesota and University of Virginia. This research used the facilities of the Canadian Astronomy Data Centre operated by the National Research Council of Canada with the support of the Canadian Space Agency.

\section{REFERENCES}

Abbott B. P. et al., 2016a, ApJ, 818, L22

Abbott B. P. et al., 2016b, Phys. Rev. Lett., 116, 061102

Adams S. M., Kochanek C. S., 2015, MNRAS, 452, 2195

Adams S. M., Kochanek C. S., Prieto J. L., Dai X., Shappee B. J., Stanek K. Z., 2016a, MNRAS, 460, 1645

Adams S. M., Kochanek C. S., Gerke J. R., Stanek K. Z., 2016b, MNRAS, preprint (arXiv:1610.02402)

Alard C., 2000, A\&AS, 144, 363

Alard C., Lupton R. H., 1998, ApJ, 503, 325

Beasor E. R., Davies B., 2016, MNRAS, 463, 1269

Belczynski K., Holz D. E., Bulik T., O’Shaughnessy R., 2016, Nature, 534, 512

Blagorodnova N. et al., 2017, ApJ, 834, 107

Botticella M. T. et al., 2009, MNRAS, 398, 1041

Botticella M. T., Smartt S. J., Kennicutt R. C., Cappellaro E., Sereno M., Lee J. C., 2012, A\&A, 537, A132

Bressan A., Marigo P., Girardi L., Salasnich B., Dal Cero C., Rubele S., Nanni A., 2012, MNRAS, 427, 127

Cannizzo J. K., Lee H. M., Goodman J., 1990, ApJ, 351, 38

Castelli F., Kurucz R. L., 2004, preprint (arXiv:astro-ph/0405087)

Chambers K. C. et al., 2016, preprint (arXiv:1612.05560)

Chen Y., Bressan A., Girardi L., Marigo P., Kong X., Lanza A., 2015, MNRAS, 452, 1068

Chini R., Hoffmeister V. H., Nasseri A., Stahl O., Zinnecker H., 2012 , MNRAS, 424, 1925

Clayton G. C., 2012, J. Am. Assoc. Var. Star Obser. (JAAVSO), 40, 539

Crause L. A., Lawson W. A., Kilkenny D., van Wyk F., Marang F., Jones A. F., 2003, MNRAS, 341, 785

Dolphin A. E., 2000, PASP, 112, 1383

Draine B. T., Lee H. M., 1984, ApJ, 285, 89

Ekström S. et al., 2012, A\&A, 537, A146

Elitzur M., Ivezić Ž., 2001, MNRAS, 327, 403

Ertl T., Janka H.-T., Woosley S. E., Sukhbold T., Ugliano M., 2016, ApJ, 818,124

Fryer C. L., Belczynski K., Wiktorowicz G., Dominik M., Kalogera V., Holz D. E., 2012, ApJ, 749, 91

Gail H.-P., Sedlmayr E., 2014, Physics and Chemistry of Circumstellar Dust Shells. Cambridge Univ. Press, Cambridge

Gerke J. R., Kochanek C. S., Stanek K. Z., 2015, MNRAS, 450, 3289

Groh J. H., Meynet G., Georgy C., Ekström S., 2013, A\&A, 558, A131

Gwyn S. D. J., 2008, PASP, 120, 212

Heger A., Fryer C. L., Woosley S. E., Langer N., Hartmann D. H., 2003, ApJ, 591, 288

Horiuchi S., Beacom J. F., Kochanek C. S., Prieto J. L., Stanek K. Z., Thompson T. A., 2011, ApJ, 738, 154

Horiuchi S., Nakamura K., Takiwaki T., Kotake K., Tanaka M., 2014, MNRAS, 445, L99

Humphreys R. M., Davidson K., 1994, PASP, 106, 1025

Iben I., Jr, Tutukov A. V., Yungelson L. R., 1996, ApJ, 456, 750

Ivanova N., Justham S., Avendano Nandez J. L., Lombardi J. C., 2013, Science, 339, 433

Ivezic Z., Elitzur M., 1997, MNRAS, 287, 799

Ivezic Z., Nenkova M., Elitzur M., 1999, preprint (arXiv:astro-ph/9910475)

Jennings Z. G., Williams B. F., Murphy J. W., Dalcanton J. J., Gilbert K. M., Dolphin A. E., Weisz D. R., Fouesneau M., 2014, ApJ, 795, 170

Jerkstrand A., Smartt S. J., Fraser M., Fransson C., Sollerman J., Taddia F., Kotak R., 2014, MNRAS, 439, 3694 
Karachentsev I. D., Sharina M. E., Huchtmeier W. K., 2000, A\&A, 362, 544 Kashi A., Soker N., 2017, MNRAS, 467, 3299

Kashi A., Davidson K., Humphreys R. M., 2016, ApJ, 817, 66

Kashiyama K., Quataert E., 2015, MNRAS, 451, 2656

Kennicutt R. C., Jr et al., 2003, PASP, 115, 928

Kochanek C. S., 2009, ApJ, 707, 1578

Kochanek C. S., 2011, ApJ, 741, 37

Kochanek C. S., 2014a, MNRAS, 444, 2043

Kochanek C. S., 2014b, ApJ, 785, 28

Kochanek C. S., 2015, MNRAS, 446, 1213

Kochanek C. S., Beacom J. F., Kistler M. D., Prieto J. L., Stanek K. Z., Thompson T. A., Yüksel H., 2008, ApJ, 684, 1336

Kochanek C. S., Szczygieł D. M., Stanek K. Z., 2012, ApJ, 758, 142

Kochanek C. S. et al., 2017, MNRAS, 467, 3347

Kreidberg L., Bailyn C. D., Farr W. M., Kalogera V., 2012, ApJ, 757, 36

Laher R. R. et al., 2014, PASP, 126, 674

Law N. M. et al., 2009, PASP, 121, 1395

Lovegrove E., Woosley S. E., 2013, ApJ, 769, 109

MacFadyen A. I., Woosley S. E., 1999, ApJ, 524, 262

MacLeod M., Macias P., Ramirez-Ruiz E., Grindlay J., Batta A., Montes G., 2017, ApJ, 835, 282

Mcley L., Soker N., 2014, MNRAS, 445, 2492

Mathis J. S., Rumpl W., Nordsieck K. H., 1977, ApJ, 217, 425

Merrill P. W., 1940, Spectra of Long-Period Variable Stars. The University of Chicago Press, Chicago, IL

Nadezhin D. K., 1980, Ap\&SS, 69, 115

O'Connor E., Ott C. D., 2011, ApJ, 730, 70

Ofek E. O. et al., 2012, PASP, 124, 62

O'Keefe J. A., 1939, ApJ, 90, 294

Özel F., Psaltis D., Narayan R., Santos Villarreal A., 2012, ApJ, 757, 55

Pan K.-C., Ricker P. M., Taam R. E., 2013, ApJ, 773, 49

Pejcha O., 2014, ApJ, 788, 22

Pejcha O., Thompson T. A., 2015, ApJ, 801, 90

Pejcha O., Metzger B. D., Tomida K., 2016a, MNRAS, 455, 4351

Pejcha O., Metzger B. D., Tomida K., 2016b, MNRAS, 461, 2527

Perna R., Duffell P., Cantiello M., MacFadyen A. I., 2014, ApJ, 781, 119

Piro A. L., 2013, ApJ, 768, L14

Prieto J. L. et al., 2008, ApJ, 681, L9

Reid M. J., Goldston J. E., 2002, ApJ, 568, 931

Reynolds T. M., Fraser M., Gilmore G., 2015, MNRAS, 453, 2885

Rodriguez J. E. et al., 2016, AJ, 151, 123

Sana H. et al., 2012, Science, 337, 444

Schlafly E. F., Finkbeiner D. P., 2011, ApJ, 737, 103

Schlegel D. J., Finkbeiner D. P., Davis M., 1998, ApJ, 500, 525

Shappee B. J., Kochanek C. S., Stanek K. Z., 2013, ApJ, 765, 150

Shiode J. H., Quataert E., 2014, ApJ, 780, 96

Smartt S. J., 2015, PASA, 32, 16

Smartt S. J., Eldridge J. J., Crockett R. M., Maund J. R., 2009, MNRAS, 395, 1409

Smith N., Li W., Filippenko A. V., Chornock R., 2011a, MNRAS, 412, 1522

Smith N., Li W., Silverman J. M., Ganeshalingam M., Filippenko A. V., 2011b, MNRAS, 415, 773

Smith N. et al., 2016, MNRAS, 458, 950

Sukhbold T., Woosley S. E., 2014, ApJ, 783, 10

Sukhbold T., Ertl T., Woosley S. E., Brown J. M., Janka H.-T., 2016, ApJ, 821,38

Thompson T. A., Prieto J. L., Stanek K. Z., Kistler M. D., Beacom J. F., Kochanek C. S., 2009, ApJ, 705, 1364

Tisserand P. et al., 2009, A\&A, 501, 985

Tylenda R., 2005, A\&A, 436, 1009

Tylenda R., Kamiński T., 2016, A\&A, 592, A134

Tylenda R., Crause L. A., Górny S. K., Schmidt M. R., 2005, A\&A, 439, 651

Ugliano M., Janka H.-T., Marek A., Arcones A., 2012, ApJ, 757, 69

Walmswell J. J., Eldridge J. J., 2012, MNRAS, 419, 2054

Webbink R. F., 1984, ApJ, 277, 355

Welch D. L., Clayton G. C., Campbell A., Barlow M. J., Sugerman B. E. K., Meixner M., Bank S. H. R., 2007, ApJ, 669, 525
Woosley S. E., 1993, ApJ, 405, 273

Woosley S. E., 2016, ApJ, 824, L10

Woosley S. E., Heger A., 2012, ApJ, 752, 32

Xiao L., Eldridge J. J., 2015, MNRAS, 452, 2597

Yusof N. et al., 2013, MNRAS, 433, 1114

Zhang W., Woosley S. E., Heger A., 2008, ApJ, 679, 639

\section{APPENDIX}

Table A1. Photometry.

\begin{tabular}{|c|c|c|c|}
\hline MJD & Filter & Magnitude & Telescope \\
\hline 51341.2 & $B$ & $23.3 \pm 0.1$ & INT \\
\hline 51341.2 & $r$ & $22.0 \pm 0.1$ & INT \\
\hline 51341.2 & $i$ & $21.4 \pm 0.1$ & INT \\
\hline 52498.9 & $V$ & $22.3 \pm 0.1$ & INT \\
\hline 52498.9 & $i$ & $21.2 \pm 0.1$ & INT \\
\hline 52935.0 & $R$ & $21.5 \pm 0.3$ & CFHT \\
\hline 52935.0 & $V$ & $21.7 \pm 0.2$ & CFHT \\
\hline 53166.8 & $3.6 \mu \mathrm{m}$ & $17.84 \pm 0.08$ & $S S T$ \\
\hline 53166.8 & $4.5 \mu \mathrm{m}$ & $17.69 \pm 0.09$ & $S S T$ \\
\hline 53260.3 & $4.5 \mu \mathrm{m}$ & $17.24 \pm 0.08$ & $S S T$ \\
\hline 53334.7 & $3.6 \mu \mathrm{m}$ & $17.42 \pm 0.05$ & $S S T$ \\
\hline 53334.7 & $4.5 \mu \mathrm{m}$ & $17.24 \pm 0.07$ & $S S T$ \\
\hline 53553.0 & $B$ & $22.8 \pm 0.1$ & CFHT \\
\hline 53553.0 & $R$ & $21.3 \pm 0.3$ & CFHT \\
\hline 53553.0 & $U$ & $23.5 \pm 0.2$ & CFHT \\
\hline 53553.0 & $V$ & $21.7 \pm 0.1$ & CFHT \\
\hline 53571.2 & $4.5 \mu \mathrm{m}$ & $17.32 \pm 0.06$ & $S S T$ \\
\hline 53571.2 & $3.6 \mu \mathrm{m}$ & $17.49 \pm 0.06$ & $S S T$ \\
\hline 53571.2 & $4.5 \mu \mathrm{m}$ & $17.35 \pm 0.07$ & $S S T$ \\
\hline 53630.8 & $4.5 \mu \mathrm{m}$ & $16.46 \pm 0.11$ & $S S T$ \\
\hline 53676.0 & $3.6 \mu \mathrm{m}$ & $17.39 \pm 0.05$ & $S S T$ \\
\hline 53676.0 & $4.5 \mu \mathrm{m}$ & $17.12 \pm 0.05$ & SST \\
\hline 53959.5 & $4.5 \mu \mathrm{m}$ & $16.98 \pm 0.07$ & $S S T$ \\
\hline 54006.2 & $3.6 \mu \mathrm{m}$ & $17.04 \pm 0.04$ & $S S T$ \\
\hline 54006.2 & $4.5 \mu \mathrm{m}$ & $16.92 \pm 0.05$ & $S S T$ \\
\hline 54065.9 & $3.6 \mu \mathrm{m}$ & $17.17 \pm 0.05$ & $S S T$ \\
\hline 54065.9 & $4.5 \mu \mathrm{m}$ & $16.90 \pm 0.05$ & SST \\
\hline 54098.0 & $3.6 \mu \mathrm{m}$ & $17.02 \pm 0.04$ & SST \\
\hline 54098.0 & $4.5 \mu \mathrm{m}$ & $16.85 \pm 0.052$ & $S S T$ \\
\hline 54285.0 & $3.6 \mu \mathrm{m}$ & $17.14 \pm 0.05$ & $S S T$ \\
\hline 54285.0 & $4.5 \mu \mathrm{m}$ & $16.94 \pm 0.05$ & $S S T$ \\
\hline 54289.2 & $\mathrm{~F} 606 \mathrm{~W}$ & $23.09 \pm 0.01$ & $H S T$ \\
\hline 54289.3 & $F 814 \mathrm{~W}$ & $20.77 \pm 0.01$ & $H S T$ \\
\hline 54324.4 & $3.6 \mu \mathrm{m}$ & $17.19 \pm 0.05$ & $S S T$ \\
\hline 54324.4 & $4.5 \mu \mathrm{m}$ & $16.97 \pm 0.05$ & $S S T$ \\
\hline 54395.9 & $3.6 \mu \mathrm{m}$ & $16.96 \pm 0.04$ & $S S T$ \\
\hline 54461.1 & $3.6 \mu \mathrm{m}$ & $16.86 \pm 0.03$ & $S S T$ \\
\hline 54461.1 & $4.5 \mu \mathrm{m}$ & $16.66 \pm 0.04$ & $S S T$ \\
\hline 54492.9 & $3.6 \mu \mathrm{m}$ & $16.93 \pm 0.04$ & $S S T$ \\
\hline 54589.4 & $B$ & $24.43 \pm 0.04$ & LBT \\
\hline 54589.4 & $R$ & $21.70 \pm 0.01$ & LBT \\
\hline 54589.5 & $U$ & $25.18 \pm 0.26$ & LBT \\
\hline 54590.4 & $R$ & $21.73 \pm 0.01$ & LBT \\
\hline 54652.4 & $B$ & $24.41 \pm 0.03$ & LBT \\
\hline 54652.4 & $U$ & $27.39 \pm 1.90$ & LBT \\
\hline 54652.4 & $V$ & $23.01 \pm 0.01$ & LBT \\
\hline 54665.8 & $3.6 \mu \mathrm{m}$ & $16.700 \pm 0.037$ & $S S T$ \\
\hline 54665.8 & $4.5 \mu \mathrm{m}$ & $16.541 \pm 0.041$ & $S S T$ \\
\hline 54761.8 & $V$ & $>18.8$ & Arbour \\
\hline 54763.8 & $V$ & $>18.6$ & Arbour \\
\hline 54795.1 & $B$ & $23.32 \pm 0.01$ & LBT \\
\hline 54795.1 & $V$ & $21.27 \pm 0.01$ & LBT \\
\hline
\end{tabular}


Table A1 - continued

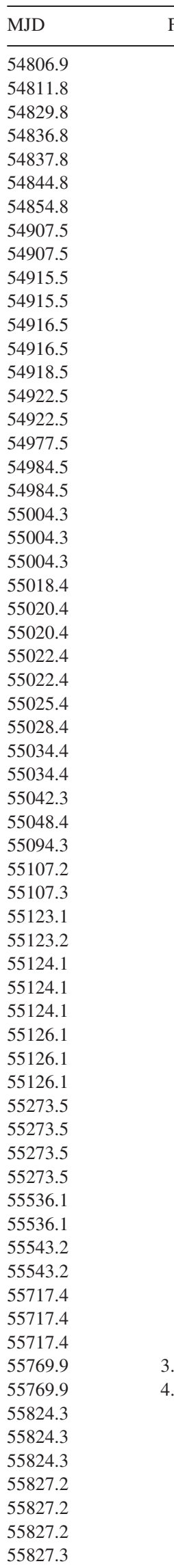

Filter

$\begin{array}{ll}V & >18.7 \\ V & >18.3 \\ V & >18.7 \\ V & >17.5\end{array}$

Telescope

Arbour

Arbour

Arbour

$>18.7$

$>17.5$

$$
>17.5
$$

$19.02 \pm 0.07$

$19.00 \pm 0.07$

$22.14 \pm 0.01$

$18.98 \pm 0.01$

$>19.6$

$19.74 \pm 0.20$

$19.82 \pm 0.13$

$19.63 \pm 0.14$

$$
>20.7
$$

$19.57 \pm 0.11$

$>19.7$

$$
>18.9
$$

$21.03 \pm 0.01$

$24.66 \pm 0.15$

$22.59 \pm 0.01$

$>20.5$

$>20.2$

$>20.2$

$>20.8$

$>20.7$

$>21.1$

$>21.4$

$>21.3$

$>21.3$

$>21.7$

$>20.6$

$>21.2$

$>20.4$

$>20.3$

$>21.4$

$$
>21.4
$$

$25.70 \pm 0.01$

$24.83 \pm 0.19$

$25.61 \pm 0.13$

$25.68 \pm 0.12$

$24.33 \pm 0.13$

$26.12 \pm 0.21$

$25.51 \pm 0.15$

$24.45 \pm 0.12$

$24.78 \pm 0.30$

$>25.97$

$26.10 \pm 0.14$

$24.93 \pm 0.10$

$24.98 \pm 0.21$

$$
>26.44
$$

$25.84 \pm 0.12$

$26.16 \pm 0.58$

$26.47 \pm 0.27$

$16.96 \pm 0.04$

$16.34 \pm 0.03$

$25.58 \pm 0.13$

$24.74 \pm 0.09$

$25.45 \pm 0.12$

$25.72 \pm 0.10$

$24.34 \pm 0.15$

$24.63 \pm 0.07$

$26.03 \pm 0.21$
Arbour

Arbour

PTF

PTF

LBT

LBT

PTF

PTF

PTF

PTF

PTF

PTF

PTF

PTF

LBT

LBT

LBT

PTF

PTF

PTF

PTF

PTF

PTF

PTF

PTF

PTF

PTF

PTF

PTF

PTF

PTF

PTF

PTF

LBT

LBT

LBT

LBT

LBT

LBT

LBT

LBT

LBT

LBT

LBT

LBT

LBT

LBT

LBT

LBT

LBT

SST

SST

LBT

LBT

LBT

LBT

\begin{tabular}{|c|c|c|c|}
\hline MJD & Filter & Magnitude & Telescope \\
\hline 55828.3 & $B$ & $25.62 \pm 0.09$ & LBT \\
\hline 55828.3 & $R$ & $24.81 \pm 0.09$ & LBT \\
\hline 55828.3 & V & $25.88 \pm 0.14$ & LBT \\
\hline 55884.1 & $R$ & $24.88 \pm 0.15$ & LBT \\
\hline 55884.1 & $B$ & $25.89 \pm 0.18$ & LBT \\
\hline 55884.1 & $V$ & $27.10 \pm 0.66$ & LBT \\
\hline 56002.0 & $3.6 \mu \mathrm{m}$ & $17.49 \pm 0.06$ & $S S T$ \\
\hline 56002.0 & $4.5 \mu \mathrm{m}$ & $16.68 \pm 0.04$ & $S S T$ \\
\hline 56045.4 & $B$ & $25.69 \pm 0.13$ & LBT \\
\hline 56045.5 & $R$ & $24.74 \pm 0.13$ & LBT \\
\hline 56045.5 & $U$ & $25.28 \pm 0.33$ & LBT \\
\hline 56045.5 & $V$ & $26.63 \pm 0.35$ & LBT \\
\hline 56090.4 & $B$ & $25.48 \pm 0.11$ & LBT \\
\hline 56090.4 & $R$ & $24.78 \pm 0.09$ & LBT \\
\hline 56090.4 & $U$ & $26.29 \pm 0.72$ & LBT \\
\hline 56090.4 & $V$ & $26.04 \pm 0.20$ & LBT \\
\hline 56093.4 & $B$ & $25.53 \pm 0.11$ & LBT \\
\hline 56093.4 & $R$ & $24.77 \pm 0.09$ & LBT \\
\hline 56093.4 & $U$ & $25.64 \pm 0.43$ & LBT \\
\hline 56093.4 & $V$ & $25.98 \pm 0.16$ & LBT \\
\hline 56215.1 & $B$ & $25.47 \pm 0.12$ & LBT \\
\hline 56215.1 & $U$ & $24.92 \pm 0.27$ & LBT \\
\hline 56215.1 & $V$ & $27.16 \pm 0.62$ & LBT \\
\hline 56217.1 & $B$ & $26.08 \pm 0.21$ & LBT \\
\hline 56217.1 & $R$ & $24.77 \pm 0.15$ & LBT \\
\hline 56217.1 & $U$ & $>25.13$ & LBT \\
\hline 56217.2 & $V$ & $>26.55$ & LBT \\
\hline 56449.4 & $B$ & $25.61 \pm 0.12$ & LBT \\
\hline 56449.4 & $R$ & $24.82 \pm 0.10$ & LBT \\
\hline 56449.4 & $U$ & $25.41 \pm 0.43$ & LBT \\
\hline 56449.4 & $V$ & $26.58 \pm 0.47$ & LBT \\
\hline 56453.4 & $B$ & $25.73 \pm 0.11$ & LBT \\
\hline 56453.4 & $R$ & $24.79 \pm 0.08$ & LBT \\
\hline 56453.4 & $U$ & $26.63 \pm 1.07$ & LBT \\
\hline 56453.4 & $V$ & $25.93 \pm 0.20$ & LBT \\
\hline 56629.1 & $B$ & $25.67 \pm 0.14$ & LBT \\
\hline 56629.1 & $R$ & $25.00 \pm 0.16$ & LBT \\
\hline 56629.1 & $U$ & $25.46 \pm 0.43$ & LBT \\
\hline 56742.6 & $3.6 \mu \mathrm{m}$ & $17.95 \pm 0.08$ & SST \\
\hline 56812.4 & $B$ & $25.57 \pm 0.11$ & LBT \\
\hline 56812.4 & $R$ & $25.05 \pm 0.15$ & LBT \\
\hline 56812.4 & $U$ & $25.29 \pm 0.35$ & LBT \\
\hline 56812.4 & $V$ & $26.40 \pm 0.32$ & LBT \\
\hline 56815.4 & $B$ & $25.93 \pm 0.15$ & LBT \\
\hline 56815.4 & $R$ & $25.00 \pm 0.10$ & LBT \\
\hline 56815.4 & $U$ & $26.20 \pm 0.76$ & LBT \\
\hline 56815.4 & $V$ & $26.00 \pm 0.16$ & LBT \\
\hline 56833.4 & $B$ & $25.73 \pm 0.14$ & LBT \\
\hline 56833.4 & $R$ & $24.82 \pm 0.10$ & LBT \\
\hline 56833.4 & $U$ & $>25.24$ & LBT \\
\hline 56833.4 & $V$ & $25.73 \pm 0.13$ & LBT \\
\hline 56836.4 & $B$ & $25.63 \pm 0.11$ & LBT \\
\hline 56836.4 & $R$ & $24.92 \pm 0.09$ & LBT \\
\hline 56836.4 & $U$ & $26.35 \pm 0.75$ & LBT \\
\hline 56836.4 & $V$ & $26.30 \pm 0.23$ & LBT \\
\hline 56839.3 & $R$ & $24.67 \pm 0.09$ & LBT \\
\hline 56839.3 & $U$ & $24.83 \pm 0.23$ & LBT \\
\hline 56839.3 & $V$ & $27.29 \pm 0.64$ & LBT \\
\hline 56900.2 & $B$ & $25.70 \pm 0.01$ & LBT \\
\hline 56900.2 & $V$ & $26.44 \pm 0.27$ & LBT \\
\hline 56916.2 & $4.5 \mu \mathrm{m}$ & $17.07 \pm 0.05$ & $S S T$ \\
\hline 56925.2 & B & $26.04 \pm 0.14$ & LBT \\
\hline 56925.2 & $R$ & $24.73 \pm 0.08$ & LBT \\
\hline 56925.2 & $U$ & $25.59 \pm 0.30$ & LBT \\
\hline
\end{tabular}

LBT

LBT

LBT
Table A1 - continued 
Table A1 - continued

\begin{tabular}{lccc}
\hline MJD & Filter & Magnitude & Telescope \\
\hline 56925.2 & $V$ & $26.01 \pm 0.14$ & LBT \\
56945.6 & $4.5 \mu \mathrm{m}$ & $17.26 \pm 0.06$ & SST \\
56981.1 & $B$ & $25.89 \pm 0.12$ & LBT \\
56981.1 & $R$ & $24.67 \pm 0.09$ & LBT \\
56981.1 & $U$ & $27.07 \pm 1.17$ & LBT \\
56981.1 & $V$ & $26.12 \pm 0.20$ & LBT \\
57132.4 & $B$ & $25.71 \pm 0.11$ & LBT \\
57132.4 & $U$ & $25.56 \pm 0.34$ & LBT \\
57132.4 & $R$ & $24.58 \pm 0.08$ & LBT \\
57132.5 & $V$ & $26.08 \pm 0.21$ & LBT \\
57163.4 & $B$ & $26.66 \pm 0.44$ & LBT \\
57163.4 & $R$ & $24.92 \pm 0.19$ & LBT \\
57163.4 & $V$ & $>26.34$ & LBT \\
57281.9 & $4.5 \mu \mathrm{m}$ & $17.50 \pm 0.08$ & SST \\
57294.0 & $4.5 \mu \mathrm{m}$ & $17.35 \pm 0.07$ & SST \\
57303.2 & UVIS $F 606 W$ & $28.44 \pm 0.46$ & $H S T$ \\
57303.3 & UVIS $F 814 W$ & $26.02 \pm 0.16$ & $H S T$
\end{tabular}

Table A1 - continued

\begin{tabular}{lccc}
\hline MJD & Filter & Magnitude & Telescope \\
\hline 57303.3 & IR F110W & $23.75 \pm 0.02$ & $H S T$ \\
57303.3 & IR $F 160 W$ & $22.38 \pm 0.02$ & $H S T$ \\
57309.1 & $B$ & $25.73 \pm 0.10$ & LBT \\
57309.1 & $U$ & $25.28 \pm 0.26$ & LBT \\
57309.1 & $R$ & $24.81 \pm 0.08$ & LBT \\
57309.1 & $V$ & $25.63 \pm 0.10$ & LBT \\
57322.1 & $4.5 \mu \mathrm{m}$ & $17.39 \pm 0.05$ & $S S T$ \\
57364.1 & $B$ & $25.56 \pm 0.08$ & LBT \\
57364.1 & $U$ & $25.33 \pm 0.25$ & LBT \\
57364.1 & $R$ & $24.84 \pm 0.11$ & LBT \\
57408.2 & $3.6 \mu \mathrm{m}$ & $18.67 \pm 0.12$ & $S S T$ \\
57408.2 & $4.5 \mu \mathrm{m}$ & $17.42 \pm 0.06$ & $S S T$ \\
\hline \multicolumn{4}{|}{}
\end{tabular}

